\title{
Building on the Ideal Free Distribution
}

\author{
Tom Tregenza
}

\section{Tregenza, T. 1995 Building on the ideal free distribution. Advances in Ecological Research 26. 253-302.}




\title{
Building on the ideal free distribution
}

\author{
TOM TREGENZA
}

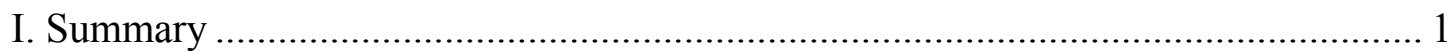

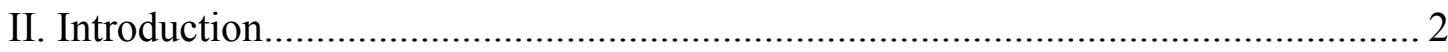

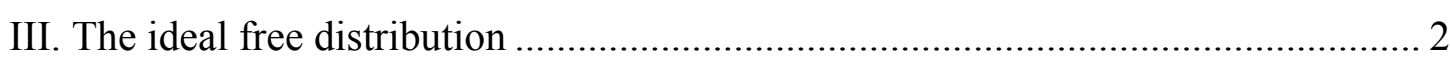

A. Ideal free distribution models .................................................................... 4

B. The basic ideal free model ................................................................. 4

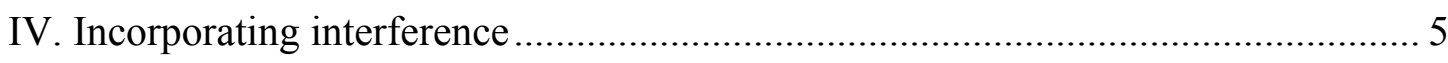

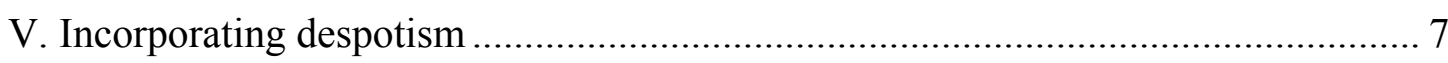

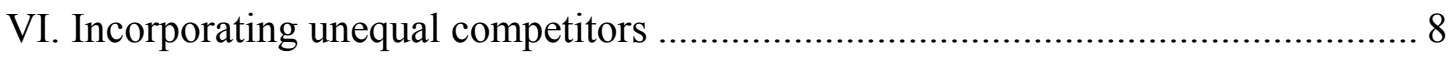

A. 2 Patch, 2 Phenotype model of phenotype-limited strategies..................... 9

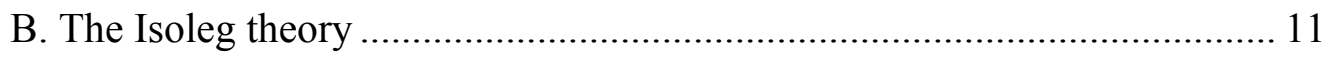

C. Continuous phenotype unequal competitor models................................. 13

D. Unequal competitor ESS distribution determined by the form of

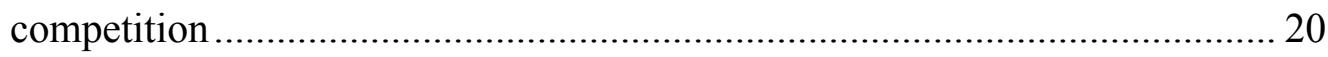

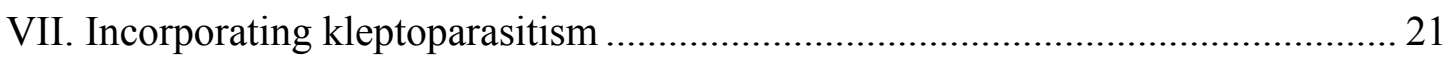

A. Modifying the basic IFD model to include kleptoparasitism ................... 21

B. A kleptoparasitic IFD model based on a functional response ................... 22

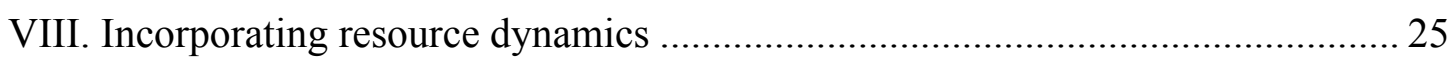

A. Continuous input models and standing crops ......................................... 25

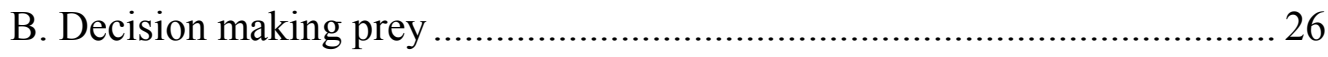

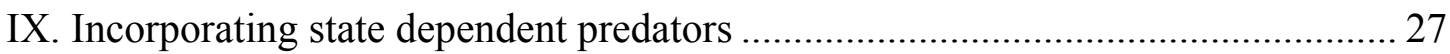

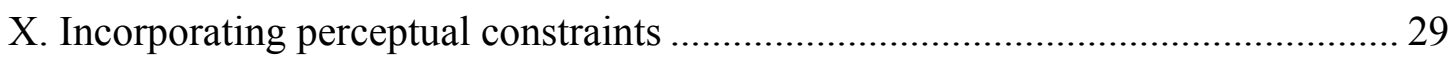

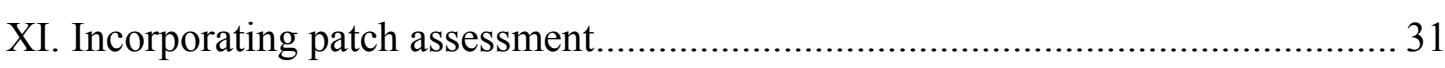

A. Patch assessment models ......................................................................... 31

B. Experimental investigations of patch assessment .................................... 37

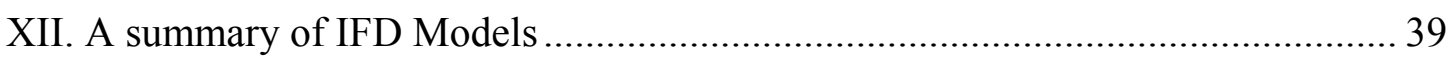

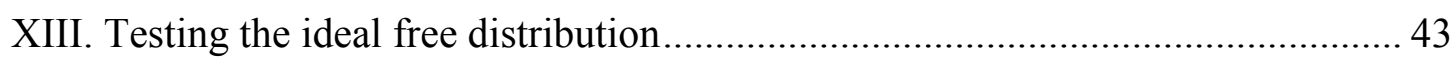

XIV. Assessing the success of ideal free distribution models.................................. 47

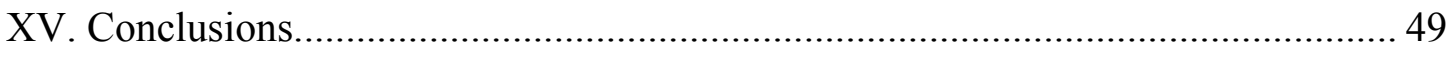

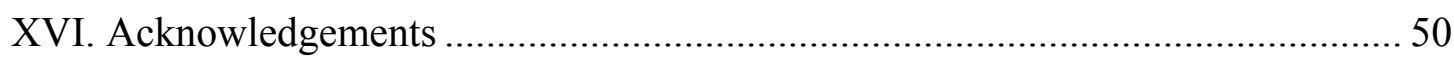

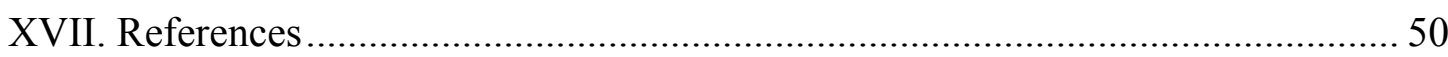


The distribution of animals around their environment is one of the cornerstones of ecology. This review aims to show how the question of animal distributions has been approached from the point of view of individual behaviour. The basis for much of the work on this subject has been the ideal free distribution theory, which considers that the suitability of any area of the environment will be a function of the density of competitors occurring there. I review how the original ideal free distribution theory has been developed and tested empirically since its inception.

The original ideal free model makes a number of assumptions, several of which have been removed by subsequent advances in the theory. The mechanisms of competition have been considered, producing two major classes of models, those dealing with exploitation competition and those which consider competition resulting from interference, including kleptoparasitism. Also, competition by resource monopolisation has been identified as requiring a separate approach. Empirical work has revealed that foragers frequently differ in their competitive abilities, inspiring a number of different theoretical advances, and a corresponding range of predictions. Other additions to the original theory are the effects of optimally distributed foragers on resource dynamics and the effect an individual's state on its optimal strategy.

Numerous empirical studies have been conducted into animal distributions, many of which have been cited as supporting the predictions of the IFD theory. However, in many cases, the evidence supporting a model is weak, indicating the need for experiments specifically designed to test the assumptions and predictions of a particular model. Experimental and field studies are reviewed and their support or otherwise for the various models is assessed. An important contribution of empirical work has been in identifying areas in which animals are unable to perfectly optimise their distribution due to constraints such as knowledge of resource distribution and ability to discern small differences in patch profitabilities. Models which incorporate patch assessment and perceptual constraints to increase the applicability of the theory are discussed. Finally, consideration is given to the success of ideal free distribution models in predicting animal distributions. 
"We can now define a habitat distribution which will provide a reference for the study of dispersive populations. This is the ideal free distribution. It rests on assumptions of habitat suitability and the adaptive state of organisms." Fretwell 1972.

Genes can equip individuals with behavioural rules which enable them to maximise competitive success through their choice of when and where to forage for resources such as food or matings. Frequently, the main variable affecting the profitability of a particular part of the environment will be the presence of other individuals also seeking to maximise their fitness.

In most studies of distribution it is assumed that animals will go to the area of the environment where their survival and reproduction will be most enhanced. Therefore an assumption is made that an individual's lifetime reproductive success is an accurate measure of the success of the genes which program it. In other words, since we are usually dealing with a large randomly mixing population the possibility that relatedness will affect distribution is excluded. Behavioural ecologists have attempted to predict the distribution resulting from each individual making an optimal decision by modelling competitor behaviour. This is essential because the microdistribution and in some cases the macro-distribution of populations is determined by individual decisions. Therefore ecological models must ultimately consider individual behaviour. The most successful result of these attempts at 'bottom up' modelling is the ideal free distribution theory. Since its inception it has been developed in a number of directions and has become one of the cornerstones of behavioural ecology.

In this review I wish to examine how the original ideal free distribution (IFD) model has been developed to include more realistic assumptions, and to examine whether the predictions of these models are supported by experimental and field studies.

\section{THE IDEAL FREE DISTRIBUTION}

The term 'ideal free distribution' was coined by Fretwell and Lucas (1970). It describes the distribution of animals which are 'ideal', meaning that they are assumed to be omniscient and to always go to the patch where their intake rate is highest, and 'free' in that they are able to enter any patch on an equal basis with residents i.e. 
without restriction or costs in terms of time or energy. This assumption demands that all animals are identical in all aspects of competitive ability. Resources are distributed in patches and are not depleted over time.

The original IFD theory predicts that animals will be distributed in a way which leads to them all experiencing equal gains even when resource patches vary in quality. The logic behind this conclusion is conceptually simple. Consider a highly competitive situation such as one in which resource items constantly arrive at a patch and individuals waiting there scramble for each item. As the number of competitors in the patch increases, the gain rate of an individual falls until it becomes so low that the animal would do better to move to a lower quality patch which is subject to less competition. This process occurs over the entire environment until an evolutionarily stable state (ESS) (Maynard Smith 1982) is reached at which point no individual can do any better by moving from its patch to another patch. Patches are exploited in proportion to the fraction of the total available resources which they receive, producing the ideal free distribution.

As well as making predictions about spatial distribution, the IFD theory can be couched in terms of time, such as predicting how long a dungfly should stay at a particular cowpat (Parker 1970), or when male butterflies ought to emerge (Bulmer 1983, Iwasa et al. 1983, Parker and Courtney 1983). The central concept is that the IFD can be used to predict the outcome of optimal decisions made by animals, where an increase in the number of animals making the same decision leads to a decrease in their individual fitnesses. This gives the IFD a potentially wide scope, with many possible applications.

Conclusions similar to those drawn by Fretwell and Lucas were also reached independently by other biologists investigating resource competition. Orians (1969) predicted that the number of females on a male's territory would be proportional to the level of resources occurring there, clearly preempting the thinking which went to make up the IFD theory. He recognised that as the best patches become crowded the average reproductive success will be expected to drop, and hence that animals in lower quality patches do not necessarily have lower fitness. In the same year, Brown (1969), studying Great tits (Parus major) came to similar conclusions. Despite couching his argument in terms of group selection theory, he recognised that "the optimal mix for maximum production requires that some individuals breed in relatively poor habitats", and that the poor habitat and the good habitat would both be exploited in proportion to their value. Parker $(1970 ; 1974)$ used identical logic to that in the IFD when he described an 'equilibrium position' of the distribution of male dungflies around cowpats where they search for females. He made the same assumptions as Fretwell and found a distribution which conforms well to that 
predicted by the IFD, with male dungflies occurring in proportion to the number of females in areas around a cowpat.

\section{A. Ideal free distribution models}

Functional models produce organizing principles which can be used to explain existing data and generate predictions for new observations and experiments. The ideal free distribution theory has been used as the basis for numerous models each addressing one or two assumptions of the original model which are clearly unjustified in many situations. There has not been a clear progression, with one assumption being removed after another to produce an increasingly accurate model of the real world. Rather, different authors have tended to use the original IFD as a basis, and have changed that aspect which they consider most wanting.

\section{B. The basic ideal free model}

In the simplest ideal free scenario, competitors scramble for as large a share of the incoming resources as possible. This might apply to fish feeding on food items drifting downstream towards them (eg Milinski 1979) or males competing for females arriving at a particular patch (eg Parker 1970). Eating or mating takes place as soon as the resource arrives at the patch, and for the purposes of modelling is assumed to be instantaneous. Individual intake rate depends only on the input rate into the patch and on how many competitors share the patch. This situation, known as 'continuous input' can be expressed algebraically as:

$$
W_{\mathrm{i}}=Q_{\mathrm{i}} / n_{\mathrm{i}}
$$

where : $\quad W_{\mathrm{i}}=$ the average gain rate in patch ' $\mathrm{i}$ '

$Q_{\mathrm{i}}=$ the input rate into patch 'i'

$n_{\mathrm{i}}=$ the number of competitors in patch ' $\mathrm{i}$ '

Since gain rates on all patches must be equal, $W_{\mathrm{i}}=Q_{\mathrm{i}} / n_{\mathrm{i}}=W_{\mathrm{j}}=Q_{\mathrm{j}} / n_{\mathrm{j}}=$ constant for all patches i,j,k, etc. This gives the 'input matching rule' (Parker 1978); $n_{\mathrm{i}}=Q_{\mathrm{i}} / c$, which predicts that the number of competitors in a patch should be proportional to the total input received by that patch. This rule has been supported by numerous studies of continuous input situations. In the field, male dungflies have been shown to match the input of females to a cowpat (Parker 1978) and grazing catfish have been found to distribute themselves such that algal growth rates in pools 
receiving different levels of light is the same (Power 1983). Numerous laboratory studies of distribution between two feeding stations (e.g. Milinski 1979; Harper 1982; Godin and Keenleyside 1984) have also supported input matching. It has also been shown that input matching can occur over time as well as space. Parker and Courtney (1983), and Iwasa et al. (1983) found that male emergence in two species of butterflies matched the mate input due to female emergence as predicted.

A complication for the simple input matching rule occurs if some resource items go unexploited or are destroyed due to increasing numbers of scrambling competitors. For instance, competing male Tiger Blue butterflies (Tarcus theophrastus) sometimes miss females, and do so disproportionately more at higher male densities (Courtney and Parker 1985). This situation can be modelled by incorporating a constant which defines the extent of the effect of increasing predators on intake rate. This constant is called the interference constant ' $m$ '. It was proposed by Sutherland (1983) specifically for the case of non-depleting interference models but can also be applied where items are arriving continuously, hence:

$$
W_{\mathrm{i}}\left(n_{\mathrm{i}}\right)=Q_{\mathrm{i}} / n_{\mathrm{i}} m \quad \text { Where } 1 \leq m \leq \infty
$$

where $m$ models the level of resource wastage as a result of interference

\section{INCORPORATING INTERFERENCE}

Although continuous input is particularly convenient for testing the basic IFD model, it is only one type of foraging situation, and one that is probably relatively uncommon in the wild. In many environments, animals search patches for dispersed prey which remain at roughly constant density. In these 'interference situations' prey are usually assumed to stay at constant density in the short-term so intake is reduced by wasting time not wasting resources. There is now no certainty that any competitors should be found in lower quality patches. The level of interference will only dictate the equilibrium distribution when time wasted in interacting with competitors reduces intake rate, reducing patch profitabilities and causing dispersal of competitors onto lower quality sites. Allowing for the effect of interference requires a term which modifies the influence of competitor density. Sutherland (1983) proposed that the 'interference constant'; ' $m$ ' be used such that:

$$
W_{\mathrm{i}}\left(n_{\mathrm{i}}\right)=Q_{\mathrm{i}} / n_{\mathrm{i}}{ }^{m} \quad \text { Where } 0 \leq m \leq \infty
$$


The level of $m$ has important consequences for competitor distribution. In contrast to continuous input situations, a patch with half the density of resources relative to the best patch is not automatically predicted to receive half the number of foragers. If $m$ is zero it will remain completely unexploited by even a large population experiencing low interference. However this is rarely the case, for instance Goss-Custard (1980) found that Oystercatchers interfered with each other by fighting and disrupting prey, and it is probably safe to assume that this is the normal situation for most foraging vertebrates.

If interference increases it will pay some individuals to move to lower quality patches where interference is lower, and hence an ideal free distribution will again result. In animals with very high levels of mutual interference, a patch with high resource density will not support twice the number of individuals as another patch with half the resources, since interference wastes so much time on the better patch.

\section{Effects on predator distribution and prey loss}

The relationship between continuous input (2a) and interference (2b) is worth noting; although the two situations are biologically different, they are mathematically identical except for the range of $m$ (Sutherland and Parker 1992). The level of $m$ will have important effects on prey distribution. In interference situations $m$ is lower so there is less incentive for the population to spill over into less profitable sites. Hence predators are more aggregated, which tends to lead to density dependent prey mortality. Where $m=1$ such as in continuous input situations without resource wastage there is a constant predator : prey ratio so mortality is density independent, and predator distribution reflects prey distribution. If $m>1$ then low density patches will be disproportionately predated, hence predation is inversely density dependent.

As a generalisation, interference situations will lead to density dependent prey loss and continuous input situations will lead to density independent or inversely density dependent prey loss (Sutherland and Parker 1992).

It is important to recognise that continuous input situations are not simply the point on a continuum of increasing interference, at which $m=1$. Continuous input situations are probably best regarded as unusual types of depleting patches, which happen to have the same dynamics as an interference situation in which $m=1$. Although Milinski and Parker (1991) describe continuous input as being high interference, many ecologists would not use the word interference in this context. There has been a certain amount of confusion regarding the unique nature of continuous input which has lead to some authors interpreting interference situations with the expectation that the number of competitors on a patch will always match the 
level of resources. A number of examples of this mistake are reviewed in Tregenza (1994).

There are two predictions implicit in the interference model which are not stated in previous reviews, and which have not always been recognised in studies attempting to apply the theory. These are: firstly, that if there is any interference at all, all patches should be used, regardless of density, and secondly that the proportion of competitors on all patches should be constant and similarly independent of density. The basis for these predictions can be seen if we rearrange the equation $Q_{\mathrm{i}} n_{\mathrm{i}}^{-m}=Q_{\mathrm{j}}$ $n_{\mathrm{j}}^{-m}$ :

$$
\begin{aligned}
& Q_{\mathrm{i}} / Q_{\mathrm{j}}=n_{\mathrm{j}}-m / n_{\mathrm{i}}^{-m}, \\
& \log \left(Q_{\mathrm{i}} / Q_{\mathrm{j}}\right) / m=\log \left(n_{\mathrm{i}} / n_{\mathrm{j}}\right)
\end{aligned}
$$

Since $Q$ and $m$ are positive constants, the ratio $n_{\mathrm{i}} / n_{\mathrm{j}}$ must also be a constant, and can never equal zero. The model does not consider animals to be indivisible units, so a situation could exist in which the poorest patch is not used because it does not provide an equal intake rate to an entire competitor. However, in large populations these integer effects should not be important.

\section{INCORPORATING DESPOTISM}

The ideal despotic distribution was proposed by Fretwell (1972), to describe any distribution in which animals showed aggressive guarding of resources, violating the 'free' assumption of the IFD. Fretwell based his ideal despotic model on the density limiting hypothesis of Huxley (1934) who envisaged a territory as a rubber disc which can be compressed but requires increasing force as it gets smaller. If the residents of a habitat make it dangerous for unsettled individuals to enter, then their average success will be lower than the habitat average, meaning they are not free. This assumption seems well justified by studies which have found that incumbent individuals have an advantage in fights (e.g. Davies 1978, Barnard and Brown 1982).

If

$$
\begin{aligned}
S_{\mathrm{i}}= & \text { the profitability of a habitat } i \text { to incumbent individuals. } \\
T_{\mathrm{i}}= & \text { the profitability of a habitat } i \text { to a newcomer. } \\
t \quad= & \text { a density dependent factor which gives the advantage of } \\
& \text { holding a territory. It increases with density since as } \\
& \quad \text { competition increases the advantage of an exclusive } \\
& \text { resource supply rises correspondingly. } 0 \leq t<1 .
\end{aligned}
$$


Then

$$
T_{\mathrm{i}}=S_{\mathrm{i}}(1-t)
$$

In contrast to non-despotic models, the IDD assumes that patches vary in their value to individuals according to whether they are incumbents or newcomers. The size of the difference in patch values is a function of the density of incumbent individuals since it is assumed that $t$ will increase with density. This assumption appears to be supported by observation, for instance, Harris (1964) showed that the centre of a lizard's territory is more vigorously defended than the edge.

If all competitors entering the habitat seek to maximise $T \mathrm{i}$ then an equilibrium will be produced in which the values of $T \mathrm{i}$ are the same in all patches. Fretwell termed this distribution the ideal despotic distribution (IDD). Although the IDD is an elegant theory, it is limited in its applicability to real situations for the same reasons as the original ideal free distribution, something Fretwell noted: "Like the ideal free distribution the ideal despotic distribution is a useful basis for discussion but because of underlying assumptions can only approximate any real situation."

Numerous authors investigating animal distributions have found evidence of despotic behaviour, and higher gain rates for competitors in better patches. Many of these results have been deemed to be evidence to support the IDD because it comes closer to predicting the distribution than the IFD, rather than because some unique prediction has been fulfilled. For instance, Andren (1990) found that in his territorial population of Jays, birds holding territories in areas dominated by Norway spruce had higher breeding success. Similarly, Patterson (1985) found that there was a higher density of fish in better quality habitats, but that some individuals were excluded from these areas. In both these studies (see also 'Testing the IFD for further examples), observation of territoriality and the lack of alternative models has lead to the IDD being cited as a valuable predictor of despotic distributions. However, the IDD is scarcely a predictive model at all. It merely states that if animals are despotic, newly arriving individuals will be forced into lower quality patches, and that the higher the density of incumbent individuals the greater will be the depression of patch quality available to new arrivals. It is obvious that resource defence will have a profound effect on predator distributions, and the question of optimal territory size is in itself a major topic of research (see Davies and Houston 1984 for review). However, the effects of territoriality on animal distributions are still poorly understood, and lack the framework for investigation which is provided for non-despotic situations by the various IFD type models. More work in this field would be very valuable, although the combination of intrinsically unequal competitors and the competitive advantage of 
holding a territory provide a formidable challenge both to modellers and to quantitative experimental work.

\section{INCORPORATING UNEQUAL COMPETITORS}

It is clear from the majority of experimental investigations (see 'testing the IFD') that differences in competitive ability between individuals are common, violating a fundamental assumption of the IFD theory. Payoff differences may persist for two main reasons (Sutherland and Parker 1985):

1. Two different strategies used by different individuals are equally successful in the long-term, but unequal in the short-term. This could occur either as a result of selection for a mixed ESS in which two strategies have the same long term fitness, but different distributions of success over the individual's lifetime. This requires that all individuals have the same long-term fitness and that payoffs are frequency dependent, so if the ESS is disturbed, selection can restore the equilibrium. Alternatively, chance events could lead to differences in short or long term success between identical competitors.

2. There is variation between individuals and long-term fitnesses are not the same. In this case individuals could be using different strategies or the same strategy. The point is that one individual is inherently a better competitor in a specific patch than another, and the poorer competitor cannot achieve equality by changing strategy.

\section{A. 2 Patch, 2 Phenotype model of phenotype-limited strategies}

This is the simplest model to consider unequal competitors, I have included it here because although it is fairly narrow in its applicability, it nevertheless makes useful predictions for a particular situation which might be used in experimental investigations into distributions. Parker (1982) considers a situation in which two alternative strategies $\mathrm{X}$ and $\mathrm{Y}$ are possible, and fitness gains per individual in each strategy are inversely proportional to the density of competitors playing that strategy. Although Parker frames his model in terms of alternative mating strategies, it is easy to see that it could equally be applied to a choice of which of two patches to exploit. This is done in Parker and Sutherland (1986), where it is applied to a continuous input foraging situation. 
It is assumed that each individual has a competitive weight $K$. The competitive weight (Parker 1982) is a measure of each phenotype's relative competitive ability. If relative payoffs remain constant, a phenotype with a competitive weight twice that of another phenotype will be expected to receive twice the payoffs in the same patch. Payoffs depend on an individual's competitive weight relative to the mean competitive weight in a patch and on the number of competitors and input rate in that patch. When using competitive weights, it is important to avoid generating a circular definition whereby the distribution is defined in terms of competitive weights and vice versa.

Phenotype $\mathrm{A}$ has a competitive weight $K_{\mathrm{Ai}}$ in patch $\mathrm{i}$ and $K_{\mathrm{Aj}}$ in $\mathrm{j}$; $\mathrm{B}$ has $K_{\mathrm{Bi}}$ in $\mathrm{i}$ and $K_{\mathrm{Bj}}$ in $\mathrm{j}$. An IFD is sought for the frequency, $P_{\mathrm{Ai}}$, of $\mathrm{A}$ phenotypes in $\mathrm{i}$, and $P_{A j}$, of A phenotypes in $\mathrm{j}\left(P_{\mathrm{Ai}}=1-P_{\mathrm{Aj}}\right)$, and similarly $P_{\mathrm{Bi}}$ and $P_{\mathrm{Bj}}$ for $\mathrm{B}$. For analysis of this model, see Parker and Sutherland (1986) Appendix 1.

If competitive weights remain constant across patches, the situation is as follows:

$$
\frac{K_{\mathrm{Ai}}}{K_{\mathrm{Aj}}}=\frac{K_{\mathrm{Bi}}}{K_{\mathrm{Bj}}}
$$

This condition generates a range of possible equilibrium distributions, in which the relative fitnesses of the two phenotypes remains equal to the ratio of their competitive weights regardless of the distribution. The consequences of this range of equilibria are discussed later in 'Testing the IFD'.

If competitive weights do not remain constant across patches, then for a given set of values there is always a unique ESS, rather than a continuum of possible distributions. At the ESS at least one of the probabilities $P_{\mathrm{Ai}}, P_{\mathrm{Bi}}, P_{\mathrm{Aj}}, P_{\mathrm{Bj}}$ must be zero. If for convenience, we define

$$
K=\frac{K_{\mathrm{Ai}}}{K_{\mathrm{Bi}}}>\frac{K_{\mathrm{Aj}}}{K_{\mathrm{Bj}}}=L
$$

Then A does $K$ times as well as B in i, but only $L$ times as well in $\mathrm{j}$, where $K>L$. There are 3 possible ESS's resulting from this situation:

(i) All A individuals go to patch i, B individuals go to both patches. This occurs when B is so numerous that some individuals do better by "overflowing" into patch $\mathrm{i}$.

(ii) All B individuals go to patch $\mathrm{j}$, A individuals go to both patches. This is the opposite of (i). 
(iii) All A go to i, all $\mathrm{B}$ go to $\mathrm{j}$ (the phenotypes remain separated at the ESS).

Thus the ESS is always "common-sense" as opposed to "paradoxical" (see Parker 1982) in the sense that A will predominate in the patch in which has the greatest competitive advantage. It must be noted that $\mathrm{i}$ is not defined as the better patch, simply the one in which A has the greater advantage over B. The details of the ESS's can be found in Parker (1982) or Parker and Sutherland (1986). They can be illustrated graphically as in Fig. 1.

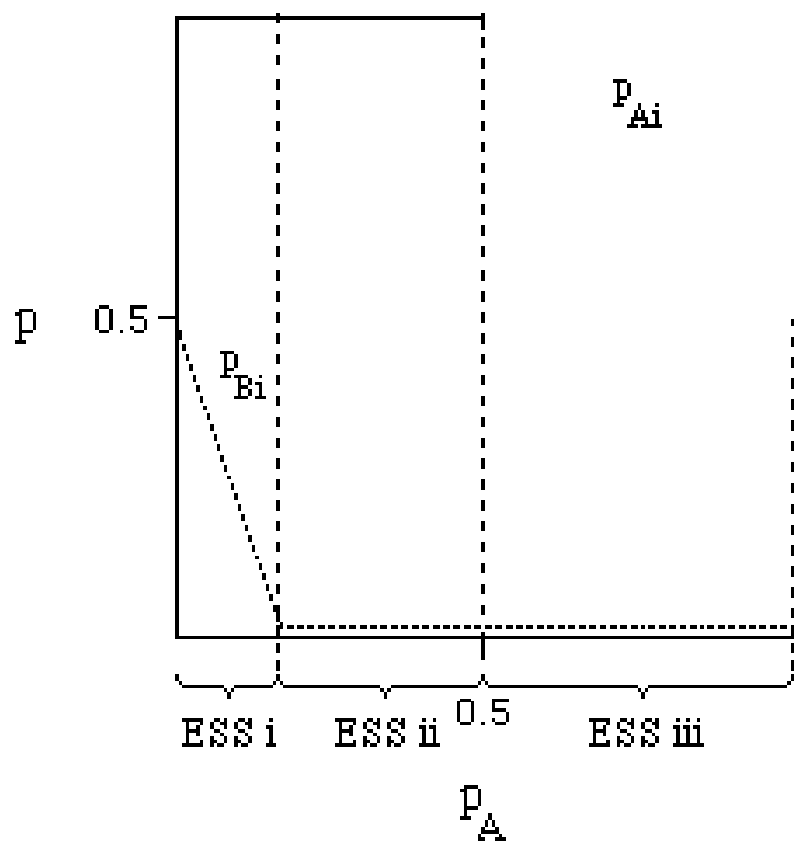

Fig. 1. The three possible ESS situations where relative payoffs change across patches. $K=5, L=1$ and $R=1$. As the frequency of phenotype $\mathrm{B}$ decreases it no longer pays it to remain in patch $\mathrm{i}$, where phenotype A has the greatest competitive advantage. Phenotype A is found in only patch i until its frequency increases to such an extent that individuals 'overflow' into patch $\mathrm{j}$. The relative fitnesses of the phenotypes are not constant but depend on the patch qualities, and on the population frequencies of the two phenotypes. After Parker (1982).

\section{B. The Isoleg theory}

A similar conclusion to that drawn from Parker's two phenotype, differing relative pay-offs model was independently reached by Rosenzweig, (1974, 1981, 1986). However, his model differs in a number of important ways. Competitors are considered to be members of different species and to have completely truncated competitive abilities. Both species prefer the richer habitat and both are affected by 
intraspecific competition. However, only the subordinate species is affected by interspecific competition, so the distribution of the dominant species is independent of that of the subordinate.

The theory allows a graph of species habitat selection to be drawn in state space. As the density of each species changes it will have implications for its own distribution and that of the subordinate species. Lines can be drawn on this graph which represent the transition points at which members of the species switch from exploiting only the good patch to exploiting both patches, and from exploiting both patches to just exploiting the poorer patch. Rosenzweig (1981) terms these lines 'isolegs' after the Greek iso - same and lego - choice. An example of an isoleg graph is shown in Fig.2.

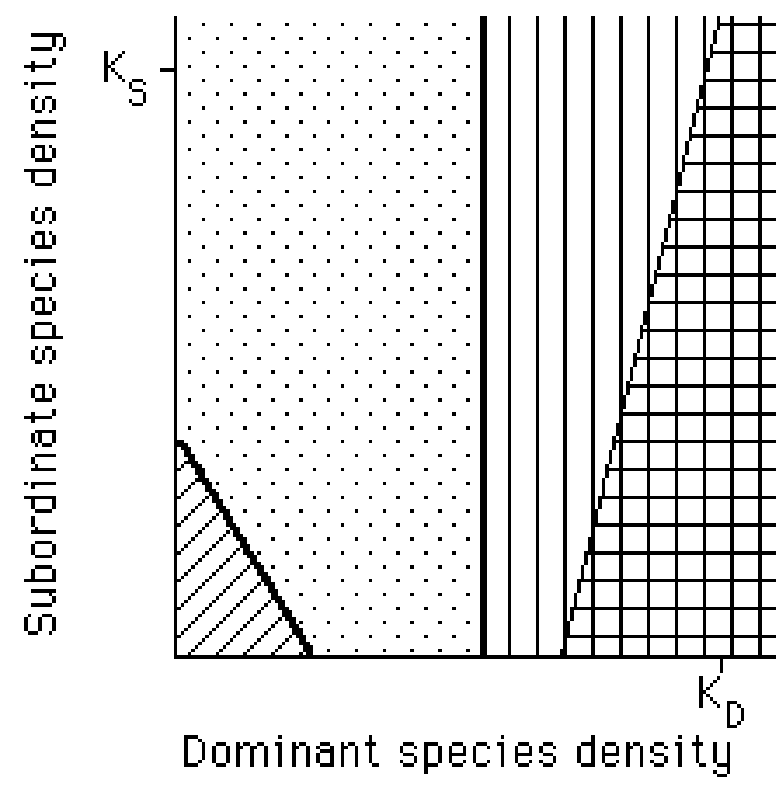

Fig. 2. Isoleg diagram of habitat selection of a dominant and a subordinate species. The subordinate has two isolegs (the thicker lines). In the diagonally shaded region all subordinates select the better habitat. In the stippled region intraspecific and / or interspecific competition leads to both patches being exploited and in the vertically shaded region all subordinates are found on the poorer patch. The dominant either uses only the good habitat to the left of the thin isoleg, or uses both patches (horizontal shading). The $\mathrm{K}$ values are the environment's carrying capacity for each species. (After Pimm et al. 1985).

Pimm et al. tested the predictions of the isoleg theory using 3 species of hummingbirds. This system is remarkably well suited to tests of habitat selection with interspecific competition since: 
1. There are two clearly truncated groups, Blue-throated hummingbirds are always dominant over Rivoli's hummingbirds and Black-chinned hummingbirds. There is apparently no dominance relationship between the two subordinate species.

2. Their food supply can be manipulated very finely since they are largely reliant on sucrose solution provided at feeders which can be controlled in value (strength of solution) and location.

3. The population can be manipulated, both by trapping birds to remove them from the population and by taking advantage of seasonal variations as they migrate into the area.

4. The abundance in relation to food resources can be estimated by using a surrogate measure, the time spent at feeders. Since feeding is overwhelmingly dominant in hummingbird time budgets during the summer observations of birds feeding at the feeders was used as an alternative to measuring local densities.

5. They are easy to identify and observe.

Observations and manipulations of the hummingbirds using two different concentrations of sucrose in feeders showed habitat selection in accordance with the predictions of the model. As Blue-throated density and/or their own density increased, Black-chinned hummingbirds switched from just using the good feeders to using both good and poor feeders. Further increases in Blue-throated's density lead to the Black-chinned's only using the lower concentration feeders. Blue throated hummingbirds switched from just feeding at the good feeders to using both with increased intraspecific density but didn't ever use only the poor patch. Even more convincingly, the Black-chinned hummingbirds switched habitats in accordance with the predicted isoleg graph. That is, the lines of equal behaviour rotated from being negative to being positive as blue throated activity increased.

\section{Continuous phenotype unequal competitor models}

As an extension to Parker (1972), Sutherland and Parker $(1985,1992)$ and Parker and Sutherland (1986), present explicit models for predator distributions with unequal competitors:

Summary of terms

Patch type

$=\quad \mathrm{i}, \mathrm{j}, \mathrm{k}$ etc. 


$\begin{array}{lll}\text { Input rate } / \text { intake rate with no interference } & =Q_{\mathrm{i}} \\ \text { Competitive phenotypes } & =\mathrm{A}, \mathrm{B}, \mathrm{C} \text { etc. } \\ \text { Total number of competitors in a patch } \mathrm{i} & =n_{\mathrm{i}} \\ \text { Competitive weights (of } \mathrm{A} \text { in i) } & =K_{\mathrm{Ai}}\end{array}$

\section{Continuous input situations}

The payoff of individual $\mathrm{A}$ in a patch i was taken to be

$$
W_{\mathrm{Ai}}=\left(K_{\mathrm{Ai}} / K_{\mathrm{i}}\right) Q_{\mathrm{i}} n_{\mathrm{i}^{-}}^{-m}
$$

Resource wastage is usually assumed to be zero for the purposes of modelling so that $m=1$.

\section{Interference situations}

In the interference situation $m$ is scaled by $\left(K_{\mathrm{Ai}} / K_{\mathrm{i}}\right)^{-1}$ this means that better competitors suffer less from interference, and hence

$$
W_{\mathrm{Ai}}=Q_{\mathrm{i}} n_{\mathrm{i}}^{-m}\left(K_{\mathrm{i}} / K_{\mathrm{Ai}}\right)
$$

Sutherland and Parker (1992) point out that the choice of which equation to use (6a or $6 \mathrm{~b})$ is in fact arbitrary, with the biological situation exerting its influence via the range and level of $m$. The main difference between the two models is that in (6a) differences in phenotype $\left(K_{\mathrm{ai}} / K_{\mathrm{i}}\right)$ affect the intake rate $\mathrm{Q}_{\mathrm{i}}$ whereas in equation $(6 \mathrm{~b})$ they scale the effect of $m$.

If logs are taken of equations (5) and (6) they can be expressed in terms of the graph equation $y=c+m x$.

$$
\begin{aligned}
& \log W_{\mathrm{Ai}}=\log \left(K_{\mathrm{Ai}} / K_{\mathrm{i}}\right)+\log Q_{\mathrm{i}}-m \log n_{\mathrm{i}} \\
& \log W_{\mathrm{Ai}}=\log Q_{\mathrm{i}}-m\left(K_{\mathrm{i}} / K_{\mathrm{Ai}}\right) \log n_{\mathrm{i}}
\end{aligned}
$$

It can be seen that in equation (7a) the phenotype differences $\left(K_{\text {ai }} / K_{i}\right)$ affect the intercept whereas in equation (7b) they affect the slope (see Fig. 3.) This difference can lead to radically different predictions of distribution. 


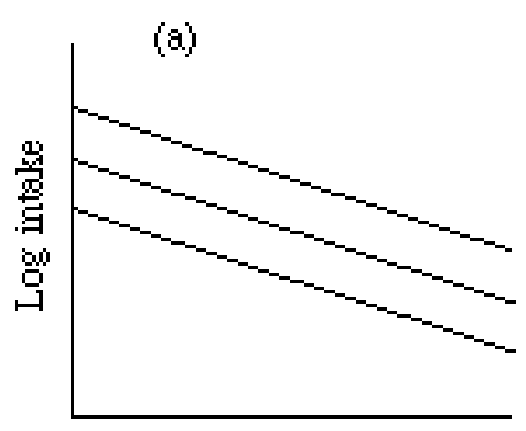

(b)

Log density competitors

Fig. 3. (a) Phenotype scales intercept, as suggested for continuous input situations or when competitors differ in search efficiency. (b) Phenotype scales slope as suggested for situations where different phenotypes suffer different levels of interference. (After Sutherland and Parker 1985).

\section{Distributions Resulting from the models}

Depending on whether competitive ability has the same effect across patches, two main types of distribution were found by Parker and Sutherland (1986) and Sutherland and Parker (1992).

\section{Equilibrium distributions}

In continuous input situations where relative payoffs remain constant across patch types no one ESS prevails. A range of distributions can occur provided that the sum of competitive weights relative to the input rate in each patch remains constant across all patches. The equilibrium attained depends on the starting condition.

\section{ESS truncated phenotype distribution}

In interference situations, patches with high resource density have more competitors and hence higher interference. The relative payoffs of an individual in a population of unequal competitors will not be constant, since better competitors will achieve their highest intake in the high resource density, high interference patches and poor competitors will do best in the low interference patches. This is because a better competitor suffers less from an increase in competitor density and hence its interference constant $m$ is a lower value than that of a poor competitor. A similar inequality can also occur in a continuous input situation if there is some extraneous reason for differential advantage across patches. For example, predators which had learnt to identify a particular type of prey would only find this advantageous in patches in which that prey type occurred.

If relative payoffs of phenotypes across patches are not equal then it is predicted that the better competitors will end up in the patches where they have the greatest advantage. In interference models this means in the best patches, in continuous input 
situations it is dependent on which patches produce the greatest relative increase in payoffs which may not be the best patches. This produces a situation in which competitors are truncated across patch types with only competitors of adjacent competitive abilities coexisting in the same patch.

No more than one phenotype can play a mixed strategy across the same two patch types adjacent in the rank order of patch qualities. The phenotypes which may show a mixed strategy ('boundary phenotypes') are the best in one and worst in the other of the two patches they exploit. This distribution is described in Fig. 4.

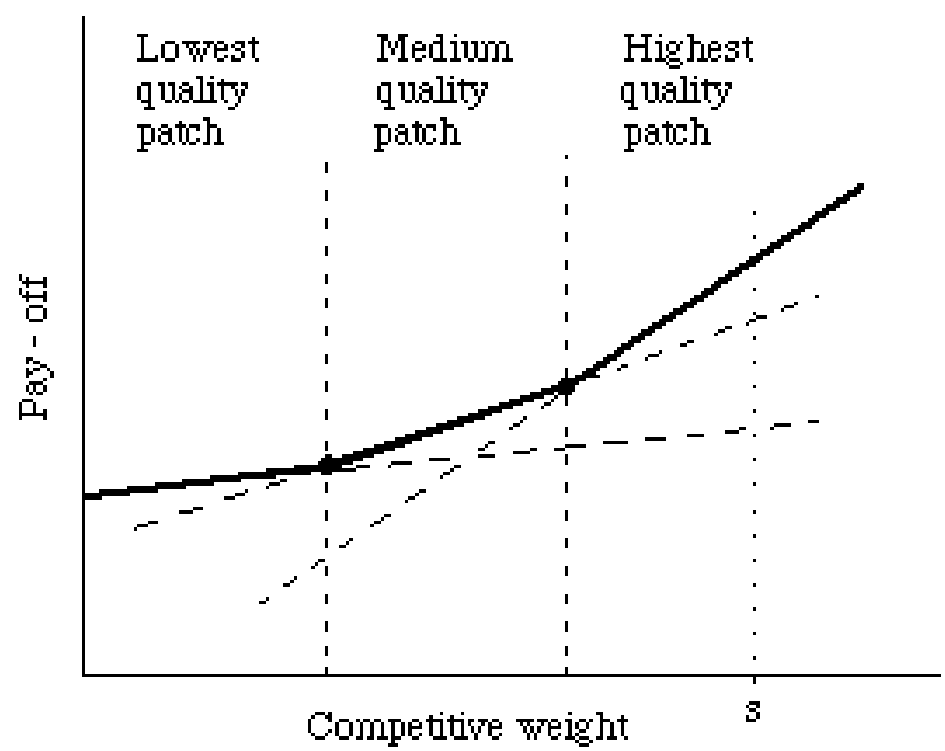

Fig. 4. The relationship between pay-off and competitive weight in three patches at the ESS (solid line). Notice that the boundary phenotypes (solid dots) have the same pay-offs in each of the two adjacent patch types. The pay-offs of mutants (dotted lines) are always lower. For instance a mutant of competitive weight ' $\mathrm{s}$ ' gains a payoff in each patch shown by the lines which intersect the vertical line at s. If it stays in the highest quality patch, it gets the payoff indicated by the solid line; if it moves to the medium patch its gains are lower, and if it moves to the lowest patch they are lower still. Therefore, we expect that different phenotypes will be truncated between patch qualities according to their relative competitive ability. (After Milinski and Parker 1991, from Parker and Sutherland 1986).

If a mutant individual arises (which is not a boundary phenotype), it will experience reduced gains if it moves to an adjacent patch, as represented by the dotted lines on the graph. If it moves to a higher input patch it will suffer because its high $m$ value means that it will be adversely affected by interference and consequently its intake will be reduced. If it moves to a lower input patch it will be unable to benefit 
sufficiently from the lack of interference to make up for the reduced input and so again its intake will fall.

\section{Differences between the two models, Intercept scaling and slope scaling} Sutherland and Parker (1992) assessed the differences between their models by first considering the conditions necessary for a given phenotype to play a pure or a mixed strategy.

In order to play a pure strategy, that is, all individuals of a particular phenotype occur on the same patch type, all individuals of phenotype $A$ must do better by playing strategy $i$ than they do by playing $j$. Given that the distribution is $¥$ and no other individual switches simultaneously:

$$
W_{\mathrm{Ai}}\left(K_{\mathrm{Ai}}, K_{\mathrm{i}}, n_{\mathrm{i}}, ¥\right)>\mathrm{W}_{\mathrm{Aj}}\left(K_{\mathrm{Aj}}, K_{\mathrm{j}}, \mathrm{n}_{\mathrm{j}}, ¥ \mathrm{Aij}\right) \quad \text { for all } \mathrm{j} \text {. }
$$

Where: $\quad W_{\mathrm{Ai}} \quad=$ The Payoff to individuals of phenotype $\mathrm{A}$ in $\mathrm{i}$.

$K_{\mathrm{Ai}} \quad=$ The competitive weight of $\mathrm{A}$ in $\mathrm{i}$.

$K_{\mathrm{i}} \quad=$ The mean competitive weight in $\mathrm{i}$

$n_{\mathrm{i}} \quad=$ The total number of competitors in patch $\mathrm{i}$.

$¥_{\text {Aij }} \quad=$ The equilibrium distribution altered by an individual of phenotype A switching from patch i to patch $\mathrm{j}$.

In order to play a mixed strategy a given phenotype A must receive the same payoffs wherever they occur. Hence the situation is:

$$
W_{\mathrm{Ai}}\left(K_{\mathrm{Ai}}, K_{\mathrm{i}}, n_{\mathrm{i}} ¥\right)=W_{\mathrm{Aj}}\left(K_{\mathrm{Aj}}, K_{\mathrm{j}}, n_{\mathrm{j}} ¥ \mathrm{Aij}\right)=\text { constant } W_{\mathrm{A}}
$$

Deviations from the mixed strategy will be penalised if

$$
W_{\mathrm{A}}>W_{\mathrm{Aj}}\left(K_{\mathrm{Aj}}, K_{\mathrm{j}}, n_{\mathrm{j}} ¥ \mathrm{Aij}\right)
$$

Where $¥_{A i j}$ is the equilibrium distribution altered by a small number of A switching from $\mathrm{i}$ to $\mathrm{j}$.

Equation (8b) ensures that $¥$ is an attractor, but not that it will be restored if a large unilateral deviation occurs, since this may lead to many phenotypes adjusting their strategies. 
In the truncated distribution only one phenotype can mix strategies across a given pair of adjacent patch types. Therefore, if $W_{\mathrm{Ai}}=W_{\mathrm{Aj}}$ (A is the boundary phenotype) then $W_{\mathrm{Bi}}>W_{\mathrm{Bj}}$ since any other phenotype which does as well as $\mathrm{A}$ in patch i must do worse in patch $\mathrm{j}$. This means that $W_{\mathrm{Ai}} / W_{\mathrm{Bi}} \neq W_{\mathrm{Aj}} / W_{\mathrm{Bj}}$, so unequal ratios of payoffs are a necessary condition for a truncated phenotype distribution. If the ratios of payoffs of more than one phenotype can be equal across the same two patches then the truncated phenotype distribution is ruled out. 
Phenotype alters intercept (based on the equations for continuous input) From equation $5 \mathrm{a}$

$$
W_{\mathrm{Ai}} / W_{\mathrm{Bi}}=\left[\left(K_{\mathrm{Ai}} / K_{\mathrm{i}}\right) Q_{\mathrm{i}} n_{\mathrm{i}}^{-m}\right] /\left[\left(K_{\mathrm{Bi}} / K_{\mathrm{i}}\right) Q_{\mathrm{i}} n_{\mathrm{i}}^{-m}\right]=K_{\mathrm{Ai}} / K_{\mathrm{Bi}}
$$

The same will apply to patch $\mathrm{j}: \quad W_{\mathrm{Aj}} / W_{\mathrm{Bj}}=K_{\mathrm{Aj}} / K_{\mathrm{Bj}}$

Therefore, if the relative competitive weights remain constant across patches $\left(K_{\mathrm{Aj}} / K_{\mathrm{Bj}}\right)=\left(K_{\mathrm{Ai}} / K_{\mathrm{Bi}}\right)$, so whatever $n_{\mathrm{i}}, n_{\mathrm{j}}, Q_{\mathrm{i}}, Q_{\mathrm{j}}$ or $m$ the payoff ratios will be equal. This shows that the truncated distribution obeying the rules of fig 1 . is impossible unless relative payoffs change across patches. An equilibrium distribution is produced since any distribution in which:

$$
Q_{\mathrm{i}} / K_{\mathrm{i}} n_{\mathrm{i}}^{m}=Q_{\mathrm{j}} / K_{\mathrm{j}} n_{\mathrm{j}}{ }^{m}=\text { constant }
$$

will be an equilibrium distribution. The input rate must be balanced by $K_{\mathrm{i}} n_{\mathrm{i}}{ }^{m}$ for all patches. If relative payoffs do not remain constant across patches only one phenotype can show a mixed strategy across two patch types and hence the truncated phenotype distribution is possible.

Phenotype alters slope, (from interference model).

From equation (6b)

$$
\begin{aligned}
& W_{\mathrm{Ai}} / W_{\mathrm{Bi}}=Q_{\mathrm{i}} \mathrm{n}_{\mathrm{i}}^{-m}\left(K_{\mathrm{i}} / K_{\mathrm{Ai}}\right) / Q_{\mathrm{i}} n_{\mathrm{i}}^{-m}\left(K_{\mathrm{i}} / K_{\mathrm{Bi}}\right) \\
& =n_{\mathrm{i}} m K_{\mathrm{i}}\left(1 / K_{\mathrm{Bi}}-1 / K_{\mathrm{Ai}}\right)
\end{aligned}
$$

The same will be true of the ratio of payoffs in patch $\mathrm{j}$, hence if competitive weights remain constant then $K_{\mathrm{Ai}}=K_{\mathrm{Aj}}=K_{\mathrm{A}}$ and $K_{\mathrm{Bi}}=K_{\mathrm{Bj}}=K_{\mathrm{B}}$. In order for more than one phenotype to mix across patch types, the payoff ratios must be equal (as shown above) i.e. $W_{\mathrm{Ai}} / W_{\mathrm{Bi}}=W_{\mathrm{Aj}} / W_{\mathrm{Bj}}$.

Therefore: $K_{i}\left(1 / K_{\mathrm{Bi}}-1 / K_{\mathrm{Ai}}\right) \log n_{\mathrm{i}}=K_{\mathrm{j}}\left(1 / K_{\mathrm{Bj}}-1 / K_{\mathrm{Aj}}\right) \log n_{\mathrm{j}}$

Since $K_{\mathrm{A}} \neq K_{\mathrm{B}}$ by definition (otherwise they would not represent different phenotypes), then in order for the payoff ratios to be equal $K_{\mathrm{i}} \log n_{\mathrm{i}}=K_{\mathrm{j}} \log n_{\mathrm{j}}$.

In order for phenotype A to mix across $i, j$. 


$$
\begin{aligned}
& Q_{\mathrm{i}} n_{\mathrm{i}}^{-m}\left(K_{\mathrm{i}} / K_{\mathrm{Ai}}\right) / Q_{\mathrm{j}} n_{\mathrm{j}}^{-m}\left(K_{\mathrm{j}} / K_{\mathrm{Aj}}\right) \\
& =\log Q_{\mathrm{i}}-m\left(K_{\mathrm{i}} / K_{\mathrm{Ai}}\right) \log n_{\mathrm{i}}=\log Q_{\mathrm{j}}-m\left(K_{\mathrm{j}} / K_{\mathrm{Aj}}\right) \log n_{\mathrm{j}}
\end{aligned}
$$

Therefore, since $K_{\mathrm{i}} \log n_{\mathrm{i}}=K_{\mathrm{j}} \log n_{\mathrm{j}}$ and $K_{\mathrm{Ai}}=K_{\mathrm{Aj}}=K_{\mathrm{A}}$ more than one phenotype cannot occur across the same two patch types at equilibrium unless $Q_{\mathrm{i}}=Q_{\mathrm{j}}$, in which case the two patches are the same anyway.

To summarise, when phenotype has the effect of scaling the slope of the equation as in the interference model, then more than one phenotype cannot mix across two patch types. This is a prerequisite for a truncated phenotype distribution, but does not ensure that one will occur. When phenotype has the effect of scaling the intercept of the equation as in the model based on a continuous input situation then a multiple mixed strategy equilibria can occur, and a truncated phenotype distribution is ruled out unless relative payoffs change across patches. Sutherland and Parker (1992) validated these conclusions by computer simulation. Using the intercept scaling models, distributions reached an equilibrium dependent on the starting conditions, the number of individuals occurring at each site depended on the distribution of phenotypes. Using the slope scaling model, the final distribution was independent of the starting conditions. In all cases a truncated phenotype distribution occurred with no more than one phenotype mixing across the same pair of patch types.

\section{Biological Application of the models}

Sutherland and Parker (1992) point out that their models show that there is no obvious reason why continuous input should give different distributions from interference, since it is the value of $m$ which changes. However, experiments using a continuous input of resources tend to produce equilibrium distributions of phenotypes whereas interference situations lead to unequal arrangements.

An explanation of this is that in interference studies intake rate declines with increasing competitor density due to differences in aggression between phenotypes leading to different levels of interference being experienced by different individuals. This is modelled by the "phenotype scales slope" model which describes the manner in which interference operates and also the resulting distributions. Continuous input studies show that differences in relative foraging abilities, such as speed and resource detection result in differences in intercept between phenotypes. Therefore the "phenotype scales intercept model" is appropriate since it predicts that no consistent relationship between phenotype and site quality will be observed. 
Sutherland and Parker (1992) recognise that there may be situations where choice of model is not obvious, for instance where fighting occurs in a continuous input situation so that some individuals obtain disproportionately less at higher resource densities. In these cases it will be necessary to consider how phenotype affects both slope and intercept. Also, there are many reasons why differences in phenotype distribution between patches may occur, such as perceptual constraints and variation in predation risk.

\section{Unequal competitor ESS distribution determined by the form of competition}

As an alternative to the above models of Sutherland and Parker, Korona (1989) presents a model for competition in which the competitive value of an individual manifests itself in contacts with other randomly chosen individuals. His model assumes that animals are omniscient and that competitive weights of phenotypes remain constant across patches. In each encounter, the chance of success of an individual $\mathrm{A}$ is given by:

$$
\text { Chance of success of } \mathrm{A}=\frac{\text { competitive weight of } \mathrm{A}}{\text { comp weight of } \mathrm{A}+\text { comp weight of adversary }}
$$

Intake rate is determined as a function of the resources per animal and the success of each individual in its conflicts with other competitors. The model predicts a unique equilibrium distribution which has three features:
a) Animals distribute themselves numerically in proportion to patch qualities.
b) Each phenotype occurs in the same proportion in each habitat.
c) The average gain for all individuals is equal in all patches.

Korona suggests that one on one competition is more likely than one individual simultaneously affecting all others, an implicit assumption in Sutherland and Parker's models. A disadvantage of Korona's model is that it can only be applied to a situation in which resources are simply divided up between all the competitors (such as continuous input). However, if prey are at roughly constant density and animals forage for them on a patch, intake rate is limited by search time and is not a simple function of competitor density alone. This assumption of Korona's model is unfortunate since his prediction that competitive ability will manifest itself through one to one contests would appear to be least likely in continuous input situations, 
where scrambles for resource items are likely to occur. The biological situations in which one on one competition is most obvious, such as oystercatchers occasionally attempting to steal mussels from each other, all tend to be interference situations, which clearly violate the other assumptions of the model. Nevertheless, Korona's model serves to illustrate the importance of checking the assumptions

\section{INCORPORATING KLEPTOPARASITISM}

\section{A. Modifying the basic IFD model to include kleptoparasitism}

In many animals one of the main sources of interference is in loss of prey items to conspecifics. Parker and Sutherland (1986) investigated this situation using a model in which there is a dominance hierarchy of phenotypes and competitors steal food from their subordinates. They assumed that there would be no differences in foraging ability. An individual's payoffs increased with the number of subordinates and decreased with the number of dominants in a patch. The simulation was run with 100 competitors and 10 ranks. It was assumed within an entire dominance rank, gains would exactly balance losses.

Two cases were investigated:

\section{Interference model}

Individuals search for resource items in patches and achieve a capture rate in patch $\mathrm{i}$ equal to $Q_{\mathrm{i}}$. In addition they experience gains and losses due to stealing from subordinates and being robbed of a proportion of their food by dominants.

The fitness of a given individual in patch $\mathrm{i}$ is therefore related its dominance rank $(D$ )

$$
W_{\mathrm{i}}(D)=Q_{\mathrm{i}}\left(c+G\left(n_{\mathrm{S}}\right)-L\left(n_{\mathrm{d}}\right)\right)
$$

in which

$$
\begin{aligned}
& c \quad=\text { each capture by the individual } \\
& G\left(n_{\mathrm{S}}\right)=\begin{array}{c}
\text { probable gains from subordinates where there are } \mathrm{n}_{\mathrm{S}} \\
\text { subordinates }
\end{array} \\
& \begin{array}{c}
\text { (nd }) \quad \text { probable losses to dominants where there are } \mathrm{n}_{\mathrm{S}} \\
\text { dominants }
\end{array}
\end{aligned}
$$

This equation was used to formulate a model in which gains and losses to self increase linearly with the number of individuals respectively above and below an 
individual in the hierarchy. $\left(G\left(n_{\mathrm{S}}\right)=a n_{\mathrm{S}}\right.$ and $L\left(n_{\mathrm{d}}\right)=a \mathrm{~d}_{\mathrm{d}}$ in which $a$ is a positive constant which is small enough to ensure that payoffs are never negative). A major factor limiting the model's applicability to real situations is the fact that it does not account for reduction in average prey intake rate due to competition; prey are reallocated according to dominance but average intake remains constant. However, it is still valuable in pointing to possible distributions of species practising kleptoparasitism and it would be very interesting to re-run this simulation using different assumptions.

\section{Continuous input model}

A fixed input of resource items $Q \mathrm{i}$ are shared between the competitors in a patch. The model is the same as in the interference case except that each individual's gains are density dependent and therefore:

$$
W_{\mathrm{i}}(D)=Q_{\mathrm{i}}\left(\mathrm{c}+G\left(n_{\mathrm{S}}\right)-L\left(n_{\mathrm{d}}\right)\right) / n_{\mathrm{i}}
$$

\section{Results}

Unless patch qualities differed considerably both models produced unstable distributions, since it pays dominants to move to patches containing subordinates but pays subordinates to move away from patches containing dominants. Payoffs did not remain constant across patches and there was no clear truncation. However, there was a tendency for better phenotypes to be found in better patches more of the time and for low ranking phenotypes to be found in all patch types. As in other models, due to density dependent gains the continuous input model was least sensitive to differences in patch qualities. In the dispersed resource model great differences in patch qualities lead to the poorer patch being avoided by all phenotypes.

Puliam and Caraco (1984) produced a similar result in a verbal model of the distribution of a subordinate and dominant individual between two patches. They assumed that individuals had a true estimate of their feeding rate in each of the four possible situations (on either patch with or without the other individual) and that an individual would only change patch if it could expect a higher feeding rate. Travel costs were taken to be negligible. Like Parker and Sutherland (1986) they found that when there is no intrinsic advantage to being together the dominant individual chases the subordinate from patch to patch.

\section{B. A kleptoparasitic IFD model based on a functional response}


A different approach to modelling competitive distributions has been suggested by Holmgren (In Press). His numerical model makes predictions about distribution between two patches using a series of transition equations which dictate the rate at which foragers switch between 3 activities; searching, handling and fighting. The basis of the model can be described graphically as in Fig. 5.

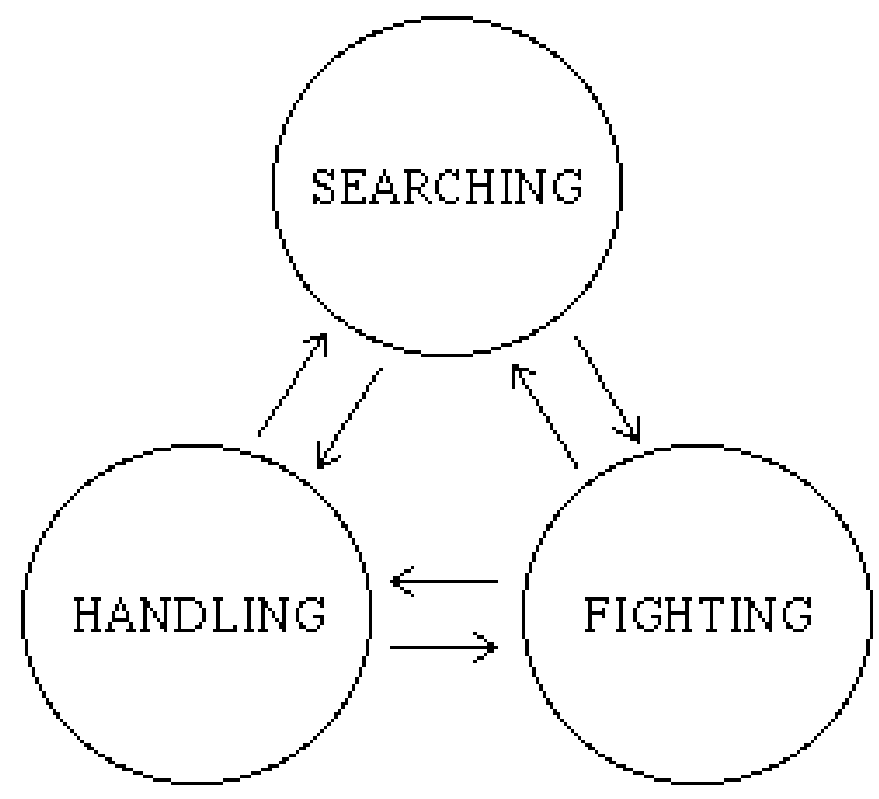

Fig. 5. Diagrammatic representation of the Holmgren model. Predators switch between 3 activities according to a set of transition equations. Searching predators may find prey and become handlers, or encounter a handling predator and begin fighting. Handling predators may be attacked, becoming fighters, or complete prey handling and resume searching. Fights take a set period of time after which the higher ranked individual begins handling and the lower ranked individual begins searching.

The rate of transition between activities is a function of the density of predators engaged in each activity, the time taken to handle each item of prey or to engage in a fight and the rate of encounters with prey. In fights between individuals the higher ranked competitor always wins. Holmgren assumes that there is no patch depletion, prey densities on the two patches are constant, irrespective of competitor density. Competition is due only to interference in the form of time lost due to fighting.

The optimal distribution was found by starting with an arbitrary distribution between patches and using an iterative procedure which always produced a stable equilibrium .

The transition rate structure of the model has two interesting advantages over other types of model. Firstly, interference is generated by interactions between 
predators, rather than being dictated by the size of a constant which must be imposed from outside. Secondly, the model is very flexible in that it can be used to investigate the effects of differences in various predator attributes such as handling efficiency, searching efficiency and dominance.

When dominance varies between competitors, dominants benefit from the presence of subordinates which do better in their absence. At low density all animals occupy the best patch, but as density increases the subordinates are the first to begin using the poorer patch. As density increases further some dominants begin using the poorer patch which is made more attractive by the presence of subordinates. This leads to an equilibrium at which both types of predator use both patch types in roughly equal proportion. With more than two predator phenotypes, a group of the most dominant individuals occurs in the best patch, with the remaining predators distributed without any relationship between relative dominance and patch quality.

When predators differ in search efficiency, but suffer equally from interference, inferior searchers are less likely to leave the high density patch since they suffer more from the increased search time associated with the poorer patch. As predator density increases the equilibrium distribution shifts from all competitors on the better patch to all the inefficient searchers on the better patch and all or a proportion of the efficient searchers on the poorer patch, presumably depending on the relative patch qualities. If a range of search efficiency phenotypes is considered there is a truncated distribution with all the best searchers occurring in the worst patch.

Variation in handling efficiency leads to less efficient predators suffering more from an increase in density since it is during prey handling that they are susceptible to kleptoparasitism. Again, at low density all predators are found on the good patch, as density increases the inefficient handlers begin utilising the poor patch. There is a density range at which the two phenotypes do not occur on the same patch followed by some of the 'efficients' moving onto the poorer patch as density increases still further. When this situation was solved for a range of phenotypes, the better handlers tended to occur on the better patch, with a second group of intermediate ability handlers occurring on the poorer patch and the remainder distributed between both.

A disadvantage with numerical simulations is that results are dependent on the parameter values used and general predictions which would allow testing are difficult to come by. An example of how this can reduce confidence in the robustness of a model's predictions comes in Holmgren's choice of patch qualities. The paper shows that equal non-depleting patch qualities (standing crops) may require very different input rates in order to be maintained. However, the choice of qualities for the two patches is one in which the poorer patch has $95 \%$ of the resources of the good patch. A larger difference in patch qualities leads to all predators aggregating in the better 
patch. Numerous field studies have found exploited prey patches varying in quality by an order of magnitude more than this (e.g. Goss-Custard 1970, Fortier and Harris 1989, Sutherland and Allport 1994). Therefore, it seems likely that this type of numerical model will be most useful as an indicator of potential qualitative effects rather than as a basis for empirical work.

\section{INCORPORATING RESOURCE DYNAMICS}

\section{A. Continuous input models and standing crops}

Because the IFD predicts the equilibrium distribution of competitors, the vast majority of theoretical work has concentrated on competition through interference or the specific case of continuous input resources. The other major source of competition between individuals, exploitation competition, does not tend to lead to a single equilibrium because as patches are depleted the optimal distribution changes. Predicting the distribution at any one time is only possible by following changes in resource levels through time.

The assumption that resources will either remain at constant density (as in interference models) or will arrive on a patch at a constant rate (as in continuous input models) places severe limitations on the IFD theory's applicability to many foraging situations. Lessells (in press) points out that interference models should be regarded as a 'snapshot' of depletion or continuous input models. She also shows that if resources are continuously input into patches, the assumption that they are immediately consumed can be relaxed by considering the standing crop of resources on each patch. The standing crop consists of those resource items which have arrived on a patch but which have yet to be consumed. If competitors are equal, all consumers must have equal consumption rates at the evolutionarily stable (ES) distribution.

If there is no interference the standing crop must be identical on each patch and hence resource mortality rate will be density independent. If interference occurs, it will decrease the intake rate more on better patches, so, since consumption rates must still be equal, there must be a greater standing crop on those patches. Prey mortality rate will be lower on better patches due to interference and will be density dependent, since patches with higher standing crops have higher competitor densities.

If there is no interference, but there is some alternative source of mortality, the losses due to this mortality will be constant across patches since all patches will have the same standing crop. Alternative mortality can lead to some patches remaining unexploited because their input rates are not sufficiently high to overcome the losses to their standing crop resulting from the alternative mortality. Those patches with 
intake rates sufficiently high to be exploited will suffer density independent mortality, due to their identical standing crops.

If there is interference, as well as some source of alternative mortality, as in a situation where some resource items are wasted (Sutherland and Parker 1992) the equilibrium distribution will depend on the relative strengths of the interrelationships between consumption rate, standing crop, alternative mortality rate and the number of competitors. The only general predictions which can be made are the same as those for a situation where there is interference but no alternative mortality.

\section{B. Decision making prey}

The patch selection models discussed so far have taken the predator's point of view and have assumed prey to be immobile. Clearly there are many examples of systems in which this is not the case, and for which consideration of prey responses is necessary. This was discussed by Sih (1984) who suggested that if a refuge from predation exists and prey are mobile that their response will dictate the determine the distribution. More recently Schwinning and Rosenzweig (1990) have modelled the distribution of populations between an open habitat and one containing refuges. They used a predator-prey system with a top predator, an intermediate predator which is eaten by the top predator and a prey item eaten by both. All individuals of all groups were assumed to be equally mobile.

Their numerical model is unlike interference IFD models or continuous input models in that competition between predators is due only to reduced intake rates resulting from prey depletion. At each time step, predator intakes are determined assuming a type II functional response (Holling 1966). However, despite basing the model on exploitation competition, prey are considered to remain at constant density, which the authors justify by assuming that habitat redistribution occurs on a much faster time scale than changes in population size. This does seem to be slightly inconsistent, since if habitat redistribution does occur on a much faster time scale than changes in population size, then one might predict that all individuals would begin foraging in the best patch and move only when it was depleted to the same level as the patch below. Nevertheless, the results may provide some insight into how kleptoparasitism affects distributions since it may be justifiable to model interference using the same equation as a functional response. Like Parker and Sutherland (1986), Schwinning and Rosenzweig found that when they ran their simulations they frequently found distributions which oscillated. A simple explanation for why this should occur is that it pays predators to move to high prey densities, whereupon it pays the prey to move elsewhere. This leads to a situation similar to that in Parker and Sutherland's kleptoparasitism models in which one type of individual constantly 
pursues another. However, through the introduction of factors not considered in Parker and Sutherland's model it was found that stabilization could be achieved by 3 means:

1. Increasing the refuge strength. The refuge habitat has a lower capture rate for both predators. If the difference between the two patches was increased the prey concentrated in the refuge, making it more attractive to the top predators compared to the open habitat. This leads to a reduced average fitness difference of the top predators between habitats, and could even lead to their extinction. Increasing the refuge strength was only stabilizing in the presence of intraspecific competition within the prey population, since without this the prey distribution is only dictated by the predator distribution, and vice versa, leading inevitably to instability.

2. Increasing intraspecific competition within the prey population. This will promote stability since the prey will tend to tolerate more predation risk and increase their presence in the open habitat due to increasingly suffering from concentration. At some point this will also allow their predators to find open and refuge habitat equally rewarding. In this case, stability increases the top predator's fitness, as it is associated with increasing prey number in the open habitat.

3. A threshold for responsiveness to fitness differences in the top predator. Stabilization can be achieved if the top predator does not respond to very small fitness differences, either because it does not perceive them, or because it ignores them. If the threshold level of the top predator was increased above the level necessary to stabilize its own distribution this subsequently stabilised the distribution of the intermediate predator and the prey. Assigning the top predator with a threshold responsiveness was found to increase its fitness slightly despite the fact that there is no cost to travel. However, it is not clear whether or not this model is very good at detecting variations in fitness, so this aspect requires further investigation.

\section{INCORPORATING STATE DEPENDENT PREDATORS}

McNamara and Houston (1990) address one of the less explicit assumptions of other IFD models; that predators seek only to maximise their short-term fitness. They point out that variability of food supply and the predation risk incurred during foraging are 
important determinants of fitness and should be considered when predicting animal distributions. This subject, known as 'risk sensitive foraging' is in itself a large field and has been comprehensively reviewed elsewhere (McNamara and Houston, 1992). Consideration is here restricted to McNamara and Houston's use of risk sensitive models to predict optimal distributions.

In their model, fitness is defined in terms of long-term survival during a nonreproductive period rather than in terms of rate of energetic gain. Animals are assigned a level of reserves, above a threshold level $L$, the animal rests, and below 0 the animal starves to death. It is assumed that animals forage continuously when their reserves are below $L$, (interruptions such as sleep are ignored). Animals may choose between two patches of food with no cost to switching patch. The behaviour of each animal at each point in time is found using a dynamic programming technique (see Ross 1983). Dynamic programming allows an animal's optimal policy at any point to be found by following it backwards through time. At each step, the animals 'state' (in this case its energy reserves) can be calculated and from this the optimal patch choice is determined. McNamara and Houston applied their model to several different combinations of patch types and both to a single individual and to a population.

Single animal, no predation, equal mean rates in both patches.

Using different patch qualities, when mean net gain is positive in both patches the low variability patch was chosen. As mean net gain decreases and becomes negative, the state region for remaining food reserves over which it is optimal to choose the high variance patch becomes larger. In effect, the animal reaches a point at which its only hope of survival is to go to the high variance patch and be lucky. Also, as mean net gains decrease, reserves decline, making the high variance patch even more likely to be chosen.

\section{Single animal, one patch is richer and is predated}

In this case, the optimal policy not surprisingly turned out to be to forage in the nonpredated patch until reserves dropped below a critical level at which point predation risk is tolerated for increased gain. When food supply is poor it determines distribution, when it is good predation takes over.

Population of consumers, no predation, variability difference between patches.

To consider the state-dependent decisions of a population of consumers, McNamara and Houston incorporate the effects of exploitation competition into their model. By assuming that food items continually appear on a patch and are found at a rate related 
to their density by the Poisson distribution. Competition manifests itself only through lower prey availability, with no interference effects.

At low prey density, all animals choose the patch with low variation. If density increases but both patches still provide positive gains, then animals with low reserves prefer the more variable patch. As density is increased still further more and more animals choose the more variable patch but not to as great an extent as with one animal since more animals on the more variable patch means that the less variable patch is a better choice. Density dependent effects are amplified as handling times decrease, hence the tendency to choose the more variable patch is negatively correlated with handling time.

\section{Population of consumers, predation risk on one patch.}

In this situation it was found that predation was very important in determining distributions at low densities and almost ignored at high densities.

McNamara and Houston point out that a state-dependent analysis of this type is appropriate for an animal which is simply trying to survive a given period of time. However, for some animals, feeding more in the present increases future reproduction, which will change the optimal policy. The distributions produced by a state-dependent IFD are a 'dynamic equilibrium', meaning that the stable situation is a result of a continuous flux of animals changing state and behaving accordingly. Therefore the model's predictions are valid only for large populations. Small populations may deviate from their predicted distribution as a result of statedependent effects but this will lead to variable and unpredictable situations.

In contrast to the simple IFD, animals making state-dependent decisions do not distribute themselves so as to equalise rates of energy gain. The fundamental assumption of the state dependent IFD, that animals will be influenced by their physiological condition as well as their long-term competitive differences is highly plausible. Therefore the possibility that animals are making state-dependent decisions must be considered when investigating distributions, since any mixed ESS is liable to be invaded by state-dependent decision makers. This has wide ranging implications for the study of competitive distributions since it is very difficult to separate statedependent and long-term competitive differences in experiments and field studies.

\section{INCORPORATING PERCEPTUAL CONSTRAINTS}

All the previous models assume that foragers are omniscient, and that their distribution must be explained through resource distribution and competition. This 
assumption is clearly unjustified in the majority of situations where animals will have an imperfect knowledge of patch profitabilities. Awareness of patch profitabilities may be limited by two factors, firstly, being unable to distinguish between the profitability of similar patches, and secondly, being unaware of the profitability of patches which have not been visited. The first problem, described as 'perceptual constraint' has been suggested as a cause for departures from an ideal free distribution. Abrahams (1986) presents a simple model in which patch suitability is defined as the total resources on the patch divided by the number of competitors present (analogous to continuous input). This is used in an iterative simulation in which a newly arriving individual chooses the patch providing the highest intake rate after the addition of a further competitor (itself). The new arrival has a perceptual limit which means that it cannot perceive differences between patch profitabilities of less than a certain magnitude. If there is more than one best patch which cannot be distinguished, the individual chooses one of them at random. After all the competitors have entered the environment, they are randomly removed from their patch and have to re-choose a patch under the same constraints. Abrahams considers this to be analogous to the patch 'switching' behaviour seen in many studies of competitive distributions. The results of the simulation confirm what one might intuitively suspect:

1. Perceptual limits can only lead to under use of better patches and overuse of poorer patches.

2. Greater perception limits lead to greater deviations from an IFD.

3. Extreme resource distributions lead to better distributions if the perception limit is low, since large differences in patch qualities mean that more animals are able to determine which is better so there are fewer 'guesses'. However, if perception limits are great then extreme resource distributions lead to a poorer fit to an IFD because guesses are 'more wrong' when there is a greater difference between patches.

Abrahams suggests two ways in which distributions resulting from perceptual constraints can be distinguished from those resulting from interference. One of these, that the perception limit model alone predicts higher intake in the better patch, only applies when competitors are equal, which is a fairly rare situation. The other utilises the fact that the perceptual constraint simulation is based on absolute numbers whereas the interference IFD uses only relative numbers. If perceptual limits are responsible for a lack of fit to the simple IFD model then decreased overall resource 
density or increased competitor numbers will lead to greater deviations since patch differences will be reduced. The interference model does not predict such an effect.

Abrahams points out that it is possible for perceptual limits and interference to operate simultaneously, and putting equations aside, this would certainly appear to be the most likely case. A range of animals have been found to be capable of complex optimal foraging behaviours, including determination of factors such as intake rate (See Stephens and Krebs (1986) for a review). It seems likely that perceptual limits will also have been reduced to a minimum, but it is inevitable that there will be a limit of some sort which will have an effect when patch profitabilities are sufficiently similar.

\section{INCORPORATING PATCH ASSESSMENT}

\section{A. Patch assessment models}

A second possible reason for violation of the assumption of omniscience is a consequence of the fact that animals cannot know the profitability of patches which have not been visited. They must either visit all the patches in their environment or must estimate patch qualities using as many sources of information as possible. There have been several attempts to model this aspect of predator behaviour, and to test whether the assumption of omniscience is necessary.

Harley, (1981) proposed that animals could decide on which patch to exploit by using a simple learning rule called the relative payoff sum (RPS) rule. The basis of the RPS rule is that an animal has a repertoire of behaviours which it can choose between. The choice of behaviour is made at a discrete time called a trial, and is decided upon by a rule which is called the animal's strategy. Harley's rule defines the probability of choosing each behaviour as a function of previous rewards. It is assumed that each trial follows a sufficiently large number of previous trials to allow the animal to have experienced different payoffs for different behaviours. Verbally, the rule states that the probability of displaying behaviour $B_{\mathrm{i}}$ at trial $t$ is equal to the sum of the previous payoffs from behaviour $B_{\mathrm{i}}$ divided by the sum of previous payoffs from all behaviours. Essentially, the animal uses a particular payoff in relation to how successful it has been in the past.

Harley adapted this basic theory to postulate a cellular mechanism by which an animal could use the RPS rule. He proposed that each behaviour has a corresponding substance which elicits it, and which is synthesised in response to the fitness increase brought by the performance of the behaviour. The substances are continuously degraded so that recent payoff information is most significant in determining 
behaviour properties. Also substances are continuously replaced to retain a residual concentration, ensuring that no behaviour can be deleted in the same way that continual degradation ensures that no behaviour can become fixed. This predicts that the probability of choosing a given behaviour is equal to the relative frequency with which it has been rewarded, i.e. it is characterised by the matching law (Herrnstein 1970).

Harley's RPS rule has been criticised by several people, most notably Houston and Sumida (1987). They present evidence that the RPS rule only matches for certain parameters, and in some situations can be bettered by a non-matching strategy. They also contend that the empirical evidence supporting the RPS is weak. This point is also made by Kacelnik and Krebs (1985) who cast doubt on Milinski (1984) and Regelmann's (1984) application of the RPS in their analysis of stickleback behaviour. Specifically, Kacelnik and Krebs show that the only piece of evidence which allowed testing of whether the goldfish were using the RPS rule rather than another rule related to the linear operator is the predicted probability of switching between patches. In the experiments the observed probability of switching was half as great as the prediction from the RPS rule. Because of these problems it must be concluded that although animals must assess their environment and base decisions on that assessment, the exact nature of the rule they use is unlikely to be identical to that proposed by Harley. Nevertheless, Harley's model has served as a pioneering heuristic device in the study of patch assessment.

Bernstein, Kacelnik and Krebs (1988) investigated the distribution of predators in a patchy environment using a decision rule similar to Harley's in that it is also based on the simple linear operator model. They point out that the ideal free distribution emphasises an equilibrium state, rather than a process; individuals move between patches until all achieve the same maximal rate of gain. Their model is based on a slightly modified version of the Sutherland (1983) model. It is best described by reviewing the derivation of this equation. Sutherland considered that in each patch consumer gain rates follow a type II functional response (Holling's (1959) disc equation):

$$
N_{\mathrm{ai}}=\frac{a^{\prime} N_{\mathrm{i}} T}{1+a^{\prime} T_{\mathrm{h}} N_{\mathrm{i}}}
$$

Where (using terminology based on application to a predator prey system)

$$
\begin{aligned}
& N_{\mathrm{i}}=\text { The number of prey present on patch } \mathrm{i} \\
& N_{\mathrm{ai}}=\text { The number of prey attacked per predator in patch } \mathrm{i}
\end{aligned}
$$




$$
\begin{aligned}
T= & \text { The total time available for searching } \\
T_{\mathrm{h}}= & \text { The time spent pursuing and handling each prey item } \\
a^{\prime}= & \text { The proportion of prey discovered by a predator/unit of } \\
& \text { search time in the absence of interference }
\end{aligned}
$$

However, predators will interfere with each other so that higher prey densities do not bring proportionally higher gain rates. This effect is described by the equation (Hassell and May 1973):

$$
a=Q P_{i}^{-m}
$$

Where: $\quad P_{\mathrm{i}}=$ the number of predators in patch $\mathrm{i}$

$Q \quad=$ the 'quest constant' (see below)

$m \quad=$ the coefficient of interference

$a \quad=a^{\prime} T_{\mathrm{S}}$ where $T_{\mathrm{S}}$ is the time actually spent searching

The quest constant $Q$ (Hassell and Varley 1969) is a constant derived from the intercept of a log graph of attack rate against predator density; as predator density increases, the attack rate decreases due to mutual interference. $Q$ represents the maximum intake rate of the predator if mutual interference is zero, and is therefore the same as the input rate in a continuous input model, for which $Q$ has previously been used (equations (1) to (7)).

The IFD theory demands that at equilibrium the number of prey captured by each predator in all patches must be the same, therefore, handling time; $T_{\mathrm{h}}$ and remaining time; $T_{\mathrm{s}}$, are constants, as are $Q$ and $N_{\mathrm{a}} / T$. This allows Sutherland (1983) to combine the two equations to show that at equilibrium:

$$
P_{\mathrm{i}}=k N_{\mathrm{i}}(1 / m)
$$

where $k$ is a constant.

Bernstein et al.'s approach differs from Sutherland's in that it does not assume an equilibrium state, but attempts to ascertain whether an equilibrium will be established using various assumptions about depletion and predator learning rate. They combine the disc equation and the Hassell and Varley model, but retain the handling time terms and instead of taking $a$, the proportion of prey discovered by a predator as a constant, they use $a^{\prime}$. This is because $a$ depends on the actual time spent searching which will be affected by the number of prey captured. Stated algebraically, $a=Q P_{1}^{-}-m$ 
whereas $a^{\prime}=Q^{\prime} P_{i}^{-m}$ where $Q^{\prime}$ is equal to $Q /$ the time actually spent searching. Holling's $a$. has the dimensions $\mathrm{L}^{2}(\mathrm{~L}=$ length) whereas Hassell and Varley's $a$ ' has the dimensions $\mathrm{L}^{2} \mathrm{~T}^{-1}$ enabling the equation to take into account that the time actually spent searching will be affected by factors such as satiation which are not otherwise accounted for.

Bernstein et al's model gives the total number of prey eaten in a patch ( $\left.N_{\text {eat }}\right)$.

Combining equations (16) and (17):

$$
N_{\mathrm{ai}}=\frac{Q^{\prime} P_{\mathrm{i}}^{-m} N_{\mathrm{i}} T}{1+Q^{\prime} P_{\mathrm{i}}^{-m} T_{\mathrm{h}} N_{\mathrm{i}}}
$$

Since $N_{\text {eat }}$ is equal to $N_{\text {ai }} P_{\mathrm{i}}$ :

$$
N_{\text {eat }_{\mathrm{i}}}=\frac{Q^{\prime} N_{\mathrm{i}} \mathrm{P}_{\mathrm{i}}^{(1-m)} T}{1+Q^{\prime} T_{\mathrm{h}} N_{\mathrm{i}} P_{\mathrm{i}}^{-m}}
$$

Where (reviewing terms)

$$
\begin{array}{ll}
N_{\text {eat }} & =\text { Total number of prey eaten in patch } \mathrm{i} \\
Q^{\prime} & =\text { A constant related to the quest constant } Q \text { (see above) } \\
T & =\text { Total time } \\
T_{\mathrm{h}} & =\text { Handling time. This is the time spent pursuing and } \\
& \quad \text { handling each prey item } \\
N_{\mathrm{i}} & =\quad \text { Total number of prey in patch } \mathrm{i} \\
n_{\mathrm{i}} & =\text { Total number of predators in patch } \mathrm{i} \\
m & =\text { The interference constant }
\end{array}
$$

Bernstein et al. used a learning rule which assumed that predators would abandon their current patch and move to a random alternative patch when local capture rate dropped below the estimated capture rate in the environment as a whole. Predators were assumed to be able to detect local capture rate precisely, but to estimate the environmental average on the basis of past experience. The relative weight of past and present capture rate on the animals' behaviour was assumed to be 
dependent on a 'memory factor', such that past experience was slowly devalued relative to present experience. This allows an animal to 'update' its estimate of average environmental profitability.

The maximum intake rate a predator can achieve is given by $T / T \mathrm{~h}$. To define a measure of instantaneous relative profitability, $q$, the number of prey captured by each predator is divided by this asymptote hence:

$$
q=\frac{N_{\text {eat }} / P}{T / T_{\mathrm{h}}} \quad \text { O } \breve{S} q \breve{S} 1, P>0
$$

$q$ is assumed to be the same for all predators occurring within a given patch it has the above limits because it is a relative measure. At the beginning of the simulation, each predator is given a personal intake rate threshold value $\gamma$. If the predator's intake rate drops below this it leaves the patch. $\gamma$ changes at each time step according to a linear operator algorithm which gives a weighted mean of immediate past and present intake rate:

$$
\gamma_{\mathrm{t}+1}=q \alpha+(1-\alpha) \gamma_{\mathrm{t}} \quad 0 \leq \alpha \leq 1
$$

Where $\gamma_{t}$ is the value of $\gamma$ at time $t$ and $\alpha$ is a memory factor.

The migrating predators are randomly distributed between patches at the beginning of the simulation. Prey are immobile. Bernstein et al. (1988) found that in the absence of depletion, and assuming that predators are equal, their model produced a distribution very close to the IFD. In fact, predators using a learning rule and migrating blindly approach an IFD more rapidly than omniscient predators which migrate only to a patch with a higher capture rate. This is likely to be an artefact of the mechanism of the simulation, in which all predators migrate synchronously, all crowding on to the better patches and immediately reducing their profitability. Nevertheless this simulation shows that even if realistic assumptions are made about predators' knowledge of their environment, optimal distributions can still be achieved.

Further manipulations to the model using depleting prey showed that prey mortality can be density dependent or independent depending on the interaction between predator efficiency and speed of learning. Rapid learning leads to density independence because predators track prey distribution. Slow learning leads to the predators' response lagging the changes in the environment such that density dependence is produced. 
Slow depletion leads to an IFD being established which in turn causes density dependent prey loss and hence the environment becomes more and more uniform. Fast depletion produces erratic effects since changes in prey distribution occur faster than learning predators can track them. Bernstein et al. (1988) suggest that this effect might lead to a systematic difference in temporal and spatial distribution between vertebrate and invertebrate predators if their learning abilities were found to be different.

Bernstein, Kacelnik and Krebs (1991) developed this model by examining the influence of travel costs and the structure of the environment, using a simple adjustment to their learning algorithm. Migration is allocated a certain number of time steps, $\tau$ during which the predator does not feed. As a consequence it updates its value of $\gamma$ according to the equation:

$$
\gamma_{\mathrm{t}+1}=(1-\alpha)^{\tau} \gamma_{\mathrm{t}}
$$

The simulation was run with varying levels of travel cost. It was found that as travel costs increase predators become reluctant to leave their current patch even if intake rate is well below what they could achieve elsewhere. This leads to predator distribution departing progressively from the IFD. Prey mortality moves from density dependence, to random, and finally to inverse density dependence. The random stage occurs because at this level of travel costs only predators in very poor habitats move patches.

The pattern of prey mortality was dependent on the scale over which changes in the environment occured. In an environment which varied progressively from good to poor or vice versa, predators gained a false impression of the environment as a whole and consequently the pattern of prey mortality was affected. Allowing random dispersal of predators, rather than movement only to neighbouring patches, increased the fit to the IFD in the same way as distributing prey at random. This model predicts that predators in a habitat which may have large systematic variations should move widely, sampling their environment in order to gain a true impression of prey distribution and hence maximise their intake.

Other recent theoretical treatments of patch estimation have focused on Bayesian updating (Oaten 1977, Green 1980, Iwasa et al. 1981 McNamara 1982). The Bayesian process involves combining past experience with current experience to estimate patch quality. These two sources of information have been called 'patch sample' and 'pre harvest' (Valone 1991). Patch sample information is accumulated 
during patch use and may include the time spent in the patch, the number of resource items obtained in the patch and the time since last resource capture. Pre-harvest information can include prior knowledge about the distribution of resources in the environment, sensory information and environmental cues that indicate patch quality. Group foragers also have access to a third source of information which has been called 'public' information (Valone 1989), since it is acquired by observation of other group members.

In Bayesian models, foragers go to the patch which their estimations lead them to believe is the most profitable one. This approach has been combined with the IFD by Cézilly and Boy (1991) who simulated a competitor distribution based on the following assumptions:

1. Individuals are free to enter either of two patches at any time.

2. Initially, individuals have no knowledge of resource distribution.

3. Switching between patches incurs a cost in terms of time.

4. Individuals are able to measure their own success and are aware of the time penalty involved in switching.

5. An individual's success in a patch relative to other competitors is proportional to its competitive weight.

6. Competitors use a Bayesian rule to assess patch qualities and choose the patch with the highest estimated profitability.

7. Relative competitive ability is the same regardless of patch.

Patches have continuous input of resource items arriving with a probability based on the Poisson distribution and with an input ratio of 2:1 between the good and poor patches. Three good, and three poor competitors were used. Good competitors had twice the competitive ability of poor ones. Because the foragers initially have no information about patch profitabilities they have an equal probability of going to either patch. It was found that under these conditions the distribution corresponded very closely to the predictions of the IFD. It was also found that better competitors tended to spend more time in the better patch and switched less frequently than the poorer competitors. However, although not mentioned by the authors, the limited number of each type of competitor used in their study means that it is inevitable that better competitors will spend more time in the good patch. This is because there are only two possible distributions which correspond to the IFD, either having all the good competitors in the good patch or having two of them and two of the poorer competitors. This gives a mean distribution in which there are 2.5 good competitors in the good patch and .5 in the poor patch. Therefore the finding that better 
competitors gain more items in the better patch may be an artefact of the simulation's limited competitor set. Nevertheless, the general conclusion provides more evidence that adaptive complex distributions of competitors can result from simple individual behaviours.

\section{B. Experimental investigations of patch assessment}

The practicalities of how foragers estimate patch quality is the subject of a growing body of research. A great deal of work has been done on solitary foragers (e.g. Hunte et al. 1985; De Vries et al. 1989; Marschall et al. 1989; Valone and Brown 1989; Cuthill et al. 1990; Valone 1991, 1992), much of which has implications for competitive systems. However, this review is restricted to studies in which animals have been observed in competitive situations.

Milinski (1984) concluded that patch choice in his three spined sticklebacks was based on individuals measuring their own foraging rate within a patch. Although this is the most simple method available, it is possible that animals could use 'short-cut' methods in order to rapidly maximise their intake whilst minimising patch sampling. Harper (1982) found that when he fed Mallards at either end of a pond at different rates an IFD was rapidly achieved. However, if he fed at equal rates, but with pieces of bread which were twice as heavy at one end then the ducks initially overused the poorer patch and took significantly longer to conform to the IFD. This suggests that initially the ducks assessed patch quality by watching the rate at which pieces of bread were thrown. Subsequently, personal intake rate assessment allowed adjustment resulting from limitations in the visual assessment method.

Croy and Hughes (1991) examined the distribution of 6 fifteen spined sticklebacks between two continuous input feeding stations. They found that like Harper's ducks, the fish were considerably more sensitive to delivery rate than they were to changes in the size of prey items. This suggests that there are certain indicators of patch quality which foragers are able to recognise more readily than others. This is to be expected, since only certain foraging cues are likely to have been present in the animal's evolutionary environment. For instance, it seems more likely that foraging fish would have the choice of two areas of a stream where food flowed past at different rates than two areas where food particles differed in size. Croy and Hughes also found that more switching occurred in the first half of each trial and that better competitors switched more frequently, enabling them to track short term changes in food profitability. Poorer competitors tended to remain at one patch and concentrate on prey missed by their superiors. This suggests that foragers differing in competitive ability may employ different patch assessment strategies. 
Animals foraging in groups have access to a source of patch information denied to dispersed foragers, that is they can use the distribution of conspecifics as an indicator of patch profitabilities. Gotceitas and Colgan (1991) investigated whether three spined sticklebacks used this shortcut. They found that when individual fish were prevented from physically sampling patches of different food availability patch choice did not conform to the IFD. Instead, individuals preferred the patch with the greatest number of conspecifics present, but only when the conspecifics were feeding. This suggests that sticklebacks initially use the presence of conspecifics to choose which patch to go to, but require physical sampling in order to choose correctly in the longer term. Pitcher and House (1987) investigated a similar question using goldfish. They found that when food was plentiful the fish divided between two equal resource patches as expected. However, when food was low the fish preferred to feed near other fish. Anti-predator advantages of group foraging were ruled out since the fish only joined groups which they could see were feeding. The authors suggest that the fish use personal intake to decide whether to remain on a patch, if intake is low they may join a group in the expectation of future increases in intake rates resulting from group foraging.

It is worth noting that rules of thumb are only valuable when foraging in certain situations. Individuals competing for resources which are patchily distributed in time as well as space will find rules of thumb useful to rapidly maximise intake whilst the distribution is being established. However, providing the number of individuals in each patch is large, once an IFD has been achieved any newly-arriving individual will do equally well by going to any patch. If the number of individuals in each patch is small then this can independently lead to selection for rules of thumb. A new arrival will do best by going to the patch with the highest number of competitors since one additional competitor will have less impact on the overall amount of resource available. It is possible that this is the reason that three spined sticklebacks choose larger groups of conspecifics.

\section{A SUMMARY OF IFD MODELS}

As we have seen, to make predictions about distributions it is necessary to ignore many factors which may frequently have important effects. Numerous models have been written in attempts to reduce the number of unjustified assumptions and come closer to reality. However, in order to keep the models sufficiently simple so as to be mathematically tractable the removal of one assumption usually has to be bought at the cost of the inclusion of another. Hence, the development of ideal free theory has not lead to an increasingly complex formula which makes fewer and fewer 
assumptions and comes ever closer to mimicking reality. Rather, a large body of work has been produced with different models tackling the removal of only one or two of the major assumptions underlying the theory. In the tables below I firstly summarise these assumptions and then consider which model deals with the removal of each. 
Table 1

Assumptions made in ideal free distribution models and likely violations of these

\section{Potential 'real' situation}

Assumption

Equal competitive abilities

Omniscience

No costs to travel between patches

No interference between competitors

Only rate of resource acquisition affects patch choice

Resources are fixed in space and time

\author{
Variation between individuals in: \\ Susceptibility to interference \\ Search efficiency \\ Handling time \\ 'State' (for instance food reserves) \\ Knowledge of resource distribution \\ Cost of travel \\ Resource holding ability \\ Susceptibility to other environmental factors \\ (e.g. predation, temperature, etc.) \\ Other priorities (e.g. mate searching, kin selection, etc.)
}

Animals may be constrained by:

Having to visit patches to assess their profitability

Inability to detect the difference between patches

Inability to remember patch profitabilities

Costs to travel between patches in terms of:

Time

Energy

Risks from predation, physical factors etc.

Various reversible factors leading to reduced intake rate with increased competitor density including:

Mutual interference - wasting time or resources

Kleptoparasitism

Despotism

Exploitation leads to patches depleting

Prey may have a dynamic distribution in response to predation

New patches may arise

Patches may have associated costs or benefits, such as:

Predation risk

Physical properties of the environment (e.g. temperature, rate of flow of moving water etc.)

Refuges, nest sites etc.

Distribution is dictated entirely by Animals may have other priorities, examples being: competitors maximising short term fitness

\section{Mate searching}

Migrations

It is evident from Table 1 that for all the key assumptions of the original IFD there are foreseeable situations in which these assumptions will be violated. Theoreticians have attempted to address this problem through the development of a range of models which are summarised in Table 2. 
Table 2

Summary of ideal free distribution models, including which of the assumptions of the original theory are removed and resulting predictions

More realistic Assumption(s)

Exploitation competition of continuous input resources leads to gains decreasing in proportion to competitor density.

As above, but resources are not consumed immediately on arrival on patch.

Competition is due to exploitation and interference.

As above, but including some alternative source of prey mortality.

Increased density leads to decreased intake rate through mutual interference.

Increased density leads to decreased intake rate through mutual interference.

Unequal competitive ability.

Increased density leads to decreased intake rate through mutual interference.

Unequal competitive ability.

Competitors steal resources from one another. Competitors vary in their dominance.

\section{Model Predictions}

Simple IFD,

Fitness of all individuals is equal on all patches.

(Fretwell and Lucas Stable equilibrium distribution of competitors 1970). with competitor density in direct proportion to input rate.

Putting resource

As above, also, standing crops of resources are dynamics into IFD models, (Lessells in press). greater in patches with higher input rates.

Putting resource

Poorer patches can remain unexploited, dynamics into IFD standing crops of resources are greater in models, (Lessells in patches with higher input rates. press).

Interference IFD, Fitness of all individuals is equal on all patches. (Sutherland 1983). The ratio of the no. of animals on patches is constant, irrespective of density. If intake rates are known at different densities, the theory can be used to predict the optimal distribution.

Phenotype alters slope of log intake vs log competitor density graph, (Sutherland and Parker 1985).

Phenotype alters intercept of $\log$ intake vs log competitor density graph, (Sutherland and Parker 1985).

Kleptoparasitism model (Parker and Sutherland 1986)
No phenotype can mix across more than two patch types.

Higher quality patches contain most competitive phenotypes. Fitness correlated with both phenotype and patch quality.

A number of stable equilibria are possible dependent on the number of animals present and their relative competitive weights. The statistically most likely equilibria is one in which no. of animals on patch is proportional to input rate (Houston and McNamara 1988)

No stable distribution, animals switch patches continuously.

Payoffs to each phenotype vary across patches. There is a weak correlation between dominance and patch quality. 
Competitors steal resources from one another. Competitors vary in their dominance.

\section{More realistic} Assumption(s)
IFD determined by the form of competition, (Korona 1989).
Each phenotype distributes itself independently in proportion to patch qualities.

\section{Model}

\section{Predictions}

\author{
Gains on a patch decrease in \\ proportion to competitor \\ density. \\ Competitors unable to \\ discriminate between \\ resource patches differing in \\ profitability less than a \\ threshold amount.
}

Predator gains increase with relative prey density due to reduced searching.

Prey move to minimise predation risk.

Increased predator density leads to decreased gains on patch due to depletion. Predators have variable energy reserves and are able to choose between patches of different variability of food supply on the basis of their risk of starvation.

As above but with predation on one patch.

Competitors steal resources from one another. Competitors vary in their dominance.

Competitors vary in their searching efficiency.

Competitors vary in their handling efficiency.

Animals must learn the profitability of different areas of the environment. Mutual interference.
Perceptual constraints model, (Abrahams 1986).

Relative to the predictions of the simple IFD (above) model predicts under-use of the better patch. This is increased when perceptual limits are great and decreased if there are large differences between patches.

3 population predator prey system,

(Schwinning and Rosenzweig 1990).

State dependent IFD (McNamara and Houston 1990). variable patch. As density increases more and more animals choose the less variable patch.
At low density, all animals choose the less

No stable distribution unless one habitat contained a strong refuge, competition between prey was strong or the predators were unable to perceive the difference between habitats of similar profitability (perceptual constraints).
State dependent IFD

A kleptoparasitic IFD based on a functional response. (Holmgren In press)

A kleptoparasitic IFD based on a functional response. (Holmgren In press)

A kleptoparasitic IFD based on a functional response. (Holmgren In press)

Bernstein, Kacelnik and Krebs, (1988).
Animals avoid the predated patch at low density but use it more and more as density increases.

Distributions change with population density. Generally, both predators use both patch types in roughly equal proportion. 
Animals must learn patch profitability and there is a time cost to travel.

Mutual interference.
Bernstein, Kacelnik There is an approximate fit to the interference and Krebs, (1991). IFD, but poorer patches are overused more if patch quality varies progressively across the environment. 
Animals switch between patches.
Houston et al. (In Press)
There is a compromise between equal intake rates on patches and equal competitor numbers. This leads to slight overuse of the poorer patch.

It is evident from the breadth of theoretical work into competitive distributions that great improvements have been made in the biological reality of IFD models. Nevertheless, the fact that each model tends to consider only a limited reduction in assumptions shows that there is still the potential for further advances.

\section{TESTING THE IDEAL FREE DISTRIBUTION}

The predictions and assumptions of ideal free distribution models have been tested in many experiments. Those prior to 1985 are reviewed by Parker and Sutherland (1986) whose table I have modified by including comment on which model explains the results of experiments and observations, (if one does). I have also added the results of work published after 1985 which was not included in their table.

Table 3

A summary of empirical investigations into competitive distributions including consideration of whether the results support any particular model

\begin{tabular}{|c|c|c|c|}
\hline Reference & Species & Result & $\begin{array}{l}\text { Support for a model of } \\
\text { predator distributions? }\end{array}$ \\
\hline
\end{tabular}

Non-continuous input situations

$\begin{array}{llll}\begin{array}{l}\text { Andren } \\ (1990)\end{array} & \text { Jay } & \begin{array}{l}\text { Highest reproductive success found in } \\ \text { dense forest, territoriality results in } \\ \text { unequal resource partitioning }\end{array} & \begin{array}{l}\text { Supports ideal despotic } \\ \text { distribution, but evidence } \\ \text { is non-quantitative }\end{array} \\ \begin{array}{l}\text { Buxton } \\ (1981)\end{array} & \text { Shelduck } & \begin{array}{l}\text { Spend less time feeding in areas of } \\ \text { dense prey }\end{array} & \begin{array}{l}\text { No convincing fit to any } \\ \text { model }\end{array} \\ \begin{array}{l}\text { Fortier and } \\ \text { Harris (1989) }\end{array} & \begin{array}{l}\text { Marine fish } \\ \text { larvae }\end{array} & \begin{array}{l}\text { Post-larval stages of copeopod } \\ \text { feeders distributed in direct } \\ \text { proportion to resources }\end{array} & \begin{array}{l}\text { May support IFD but } \\ \text { intake rates not measured. }\end{array}\end{array}$


Reference Species $\quad$ Result

Support for a model of predator distributions?

Goss-Custard Oystercatcher et al.(1982)

\author{
Goss-Custard Oystercatcher \\ et al. (1984) \\ Goss-Custard Oystercatcher \\ et al. (1992)
}

$\begin{array}{ll}\begin{array}{l}\text { Jakobsen and } \\ \text { Johnsen } \\ (1987)\end{array} & \begin{array}{l}\text { Daphnia } \\ \text { pulex }\end{array} \\ & \\ \begin{array}{l}\text { Korona } \\ (1990)\end{array} & \text { Flour beetles }\end{array}$

Messier et al. Muskrat (1990)

Møller (1991) Passerine birds

Monaghan Herring Gull
(1980)

Morris (1989) White-footed Mice

Nishida Coreid Bugs

Perusse and Feral Pigeons Lefebvre (1985)

Power (1983, Loricariid 1984) Catfish

Sibly and McCleery Herring Gull (1983)
Greater spreading out across patches occurred as density increased. Also, immatures moved off better patches. As birds matured they used better patches

Average food intake differs consistently between mussel beds

Greater spreading out across patches occurred as density increased

Both food patches depleted to same level. Proportionally more use of better patch at lower food density

With low travel costs the number of ovipositing beetles corresponded to the amount of flour on each patch

Different survival rates between patches. Some individuals gain higher fitness via resource monopolisation

In species where patch size correlates with reproductive success, younger individuals found in smaller patches.

Average food intake about five times higher in better areas of rubbish tip

Fitness of mice (by various measures) was not significantly different between three habitats

Males in bigger aggregations had higher mate acquisition probability despite identical competitive ability

Smaller food patches lead to greater dispersal across patches. Large patches lead to grouped sequential exploitation

Growth rate of algae in sunny and shaded pools is equal as a result of foraging by catfish

Average food intake consistently higher on open tip than elsewhere
Conflicts with IFD prediction that all patches should be use regardless of density. Competitors unequal.

Consistent with unequal competitor models

Conflicts with IFD prediction that all patches should be use regardless of density

Conflicts with IFD prediction that all patches should be use regardless of density

May support IFD but intake rates not measured

Consistent with ideal despotic distribution

Supports unequal competitor (truncated distribution) models

Consistent with unequal competitor models

Weak support for IFD but individual differences not measured

No support for any model, may be due to perceptual constraints

Conflicts with IFD prediction that all patches should be used regardless of density

Consistent with IFD predictions

Consistent with unequal competitor models 
Reference Species Result

Support for a model of predator distributions?

\begin{tabular}{|c|c|}
\hline $\begin{array}{l}\text { Sutherland } \\
\text { (1982) }\end{array}$ & Oystercatcher \\
\hline $\begin{array}{l}\text { Talbot and } \\
\text { Kramer } \\
(1986)\end{array}$ & Guppies \\
\hline $\begin{array}{l}\text { Thompson } \\
\text { (1981) }\end{array}$ & Shelduck \\
\hline $\begin{array}{l}\text { Thompson } \\
\text { (1984) }\end{array}$ & $\begin{array}{l}\text { Lapwing and } \\
\text { golden plover }\end{array}$ \\
\hline
\end{tabular}

Average food intake differs between parts of cockle bed

Proportion of fish in a habitat correlated with food supply, but no fit to IFD

Time spent feeding similar between areas with different prey densities

Rate of food intake greater in fields where prey is most abundant. Also, increase in density led to a change in proportional use of patches

Zwarts and Drent (1981)

Oystercatcher

Food intake constant in years of different mussel availability

\section{Continuous input situations}

Alatalo et al. Black Grouse (1992)

\section{Courtney and Tiger blue} Parker (1985) butterfly

Croy and Hughes

(1991)

Davies and Halliday (1979)

van Duren and Glass (1992)

Gillis and Kramer (1987)

Godin and Keenleyside (1984) Cichlid fish

Fifteen-spined Stickleback

Toads

Cod

Zebra fish

Cichlid fish

Mallard
Males in larger leks gain more mates. Poorer males go to smaller leks

Density of mate searching males on different sized bushes fits ideal free better than random distribution

Fish distributed in proportion to food when rate of input was source of patch variation.

Density of mate searching males in pond and edge consistent with obtaining equal numbers of females

Fish spent more time in better food patch than IFD prediction, only better competitors did better there. poorer competitors gained very little food

Deviations from IFD seen only at high densities - therefore not due to despotism, but to interference increasing with density

Average food intake same in two sites, despite individual differences

Average food intake same in two sites, despite individual differences
Consistent with unequal competitor models

No convincing fit to any model, may be due to poor patch assessment

No convincing fit to any model, may be due to poor patch assessment

No convincing fit to any model, may be due to poor patch assessment or to unequal competitors

Weak support for IFD
Supports unequal competitor models

Consistent with IFD but individual differences not studied

Supports unequal competitor models - size differences in success

Consistent with unequal competitor models - size differences in success

Significant deviation from unequal competitor IFD may be due to few competitors and very low success of poorer competitors

Supports 'ideal interference distribution' ${ }^{1}$. Conflicts with IFD models due to change in proportional patch use with density.

Supports unequal competitor models

Supports unequal competitor models 


\section{Reference Species Result}

Support for a model of predator distributions?

Inman (1990) Starlings

Järvi and

Pettersen

(1991)

Lamb and Wood-ants

Ollason

(1993)

Milinski Stickleback

(1979, 1984,

1986)

Parker (1970, Dungflies

1974)

Patterson Cichlid fish

(1985)

Sutherland et Goldfish

al. (1988)

Thornhill Lovebug

(1980)

Whitham

Aphids

(1980)
Over-use of better food patch. Unequal relative payoffs, birds more likely to be found on patch where their success is highest

Mature parr are superior competitors. Under-use of better food patch, even allowing for unequal competitive abilities.

The number of ants using continuous input sucrose patches reflected the relative input rates to two sites.

Average food intake same in two sites, despite individual differences. Ratio of phenotypes in each patch is the same as in the environment as a whole

Average mating success with newly arriving females same in different sites, but large males more successful at take-overs, which occur mainly in one site

Territorial. Higher density in preferred habitat. However, poorer habitat used before density in preferred habitat increases during introduction of fish.

Mean rank of fish inversely proportional to number of fish in site but fish gained more food in better site. No relationship between rank and time in better site found.

Larger males at bottom of swarm, smaller males at top. Mating success increases with size and position in swarm

Average number of offspring per individual equal on different sized leaves despite individual differences
No support for any model, may be due to despotism

No support for any model. May be due to perceptual constraints, incorrect assessment of competitive abilities or despotism

Supports equal competitor IFD

Supports unequal competitor models

Supports unequal competitor models, bigger males more successful and tend to occur on dropping (Borgia 1980)

Supports ideal despotic distribution

Supports unequal competitor models, deviation may be due to sampling or perceptual constraints

Supports unequal competitor models

Supports unequal competitor models

Note

1. Gillis and Kramer suggest that the term 'ideal interference distribution' should be used to describe deviations from the IFD due to various factors associated with high predator densities or 
despotism. They do not suggest any specific model associated with this name. 


\section{ASSESSING THE SUCCESS OF IDEAL FREE DISTRIBUTION MODELS}

It is clear from studies of animal distributions that different situations are best described by different IFD models. This could be taken as evidence that each situation is different and must be explained differently, or that so far, no model has been written which makes realistic assumptions about predator behaviour. In the right hand column of Table 3, I have noted only the most simple model which explains the results of the experiment, not all those which do so. Some experiments have been designated as 'consistent' with a particular theory, whereas others are described as showing 'support'. The difference between these two categories is not clear cut. I have considered that an experiment or study cannot be deemed to support a model (by which I mean the predictions of a model) unless it fulfils some quantitative prediction of the model which is distinct from those of any other model. It is obvious from the table that no single model explains all the results, however, models with the assumption that competitive abilities vary are clearly more widely applicable.

An interesting feature of continuous input IFD experiments is that many have produced distributions which conform to the equal competitor model's numerical predictions, despite the fact that the participants differed in their competitive ability and intake rates. A simple competitive weights model, (one where ratios of phenotypic payoffs stay constant across patches), would predict a range of potential equilibria, comprising all distributions in which the ratio of competitive weight to resource input in each patch was constant. Only in one of these equilibria does the average pay-off in each patch remain constant: that in which the ratio of different competitor phenotypes is the same in each patch and the habitat as a whole. The fact that this distribution was apparently over-represented suggests that there is some reason why distributions of this type are more likely to occur than any of the other equilibria.

A number of suggestions have been made to explain this situation:

1. Sutherland and Parker (1985) pointed out that the distribution most frequently seen in experiments is the most likely outcome if the equilibrium is selected by chance. They later refined the idea (Parker and Sutherland 1986) postulating that competitors distribute themselves randomly with respect to phenotype, and match numbers with input rate. This could occur if individuals were quick at assessing the input rate of a patch and the competitor density but much poorer at assessing their relative competitive weight.

Houston and McNamara (1988) analysed this theory in more detail using 
an approach based on statistical mechanics and found that the average of all potential distributions gave a weighted mean pay-off to better and poorer patches of $1.54: 1.45$. This means that for large populations there will be a slightly higher proportion of good competitors in the better location than in the habitat as a whole, rejecting Parker and Sutherland's idea except for small groups. However, Milinski and Parker (1991) point out that in practice, experiments will probably not be able to distinguish between $1.54: 1.45$ and 1.5 $: 1.5$, so the possibility that the observed distributions are a result of statistical probabilities remains.

2. Competitors could distribute themselves with respect to their own competitive rank only, so that each rank is distributed in an ideal free fashion ignoring all other ranks (N.B. Davies quoted in Milinski and Parker 1991).

3. Korona (1989), reviewed earlier, provides a third possibility. His model produces a unique ESS distribution in which each phenotype occurs in the same proportion in each habitat, as is frequently observed. Also, his model does not require that animals use a 'short-cut' patch assessment method to explain their distribution. The simplest way in which an optimal distribution could be reached is via competitors assessing their personal intake rate and moving to the patch where it is highest. This method might be superseded by a direct patch or competitor assessment method such as is suggested in the previous two explanations. However, although it is likely to allow more rapid intake rate maximisation, there is no guarantee that a short-cut assessment method will have evolved. Also, there are some situations in which patch assessment without sampling will be difficult. However, despite these advantages there are some grounds for caution when applying Korona's model which are discussed earlier.

4. Holmgren (in press ) predicts a number of alternative distributions, depending on how competitors vary in their competitive ability. His simulations predict that if they differ in dominance then an equilibrium similar to that most frequently observed will be produced. Like Korona's model, Holmgren does not invoke any more sophisticated environmental assessment than personal intake rate.

Reviewing tests of the IFD also reveals other areas in which departures from the predictions of any of the models are common. Territoriality clearly requires a 
different theoretical framework, something I have discussed earlier. Also, perceptual constraints, both environmental and psychological are likely to be a major factor in violating the assumption that competitors will always go to the patch where their intake rate is highest.

In a recent paper, Kennedy and Gray (1993) state that, "in its current form, the IFD does not accurately predict the distribution of foraging animals". Their review criticises much past work on competitive distributions and claims to provide a new perspective on deviations from the IFD theory's predictions. However, as Milinski, (in press ) points out, their paper contains a series of misconceptions and most of what they consider to be new insight has been in the literature for many years.

Kennedy and Gray claim that the IFD predicts that the proportion of competitors in a patch will match the proportion of resources occurring there. As we have seen, this is only the case for a situation in which the interference constant $m=1$, and does not apply to the majority of natural foraging scenarios. They also claim that their reanalysis of data from past distribution studies is a new finding since it shows that there is consistent overuse of poorer patches relative to the predictions of simple IFD models ('undermatching'). However, some time ago Regelmann (1984) predicted overuse of the poor patch as a result of sampling, and Abrahams (1986) incorporated this into ideal free models. Further, contrary to their claims, Kennedy and Gray's method of detecting overuse of the poorer patch is not novel, having been used by Fagen (1987).

Kennedy and Gray discuss possible reasons for undermatching. Because they expect that competitors will automatically match resources even without interference, they incorrectly predict that as interference increases there will be more overuse of the better patch (overmatching). This is contrary to the predictions of the ideal free distribution in an interference situation, where increasing interference leads to the distribution shifting from all competitors being found on the best patch with zero interference to undermatching with very high $(m>1)$ interference. I will not review all the errors in Kennedy and Gray's brief review, but would refer the reader to Milinski (in press ).

\section{CONCLUSIONS}

For decades, ecologists have described animal distributions without a comprehensive understanding of how they occur at an individual level. In the last twenty five years attempts have been made to remedy this situation through investigation of the behavioural mechanisms which ultimately lead to population distributions. The basis for much of this work has been the concept that the most important factor affecting 
the suitability of any particular part of the environment is the presence of other competitors. The original ideal free distribution theory has been refined and expanded in many directions through a large body of theoretical and experimental work. This has revealed both the complexity inherent in competitive distributions and the potential for individual behaviours to have significant effects at a population level.

The assumptions of equal competitive ability, omniscience, movement without costs and a single type of competition have all been shown to be inappropriate in many situations. When unequal competitors are considered, a range of new distributions are predicted including phenotypes being truncated across patches of different quality. Lack of omniscience and costs to movement tend to lead to greater use of better patches, but the magnitude of the effect is dependent on the composition of the environment. Several different forms of competition have been suggested, each having different effects on population distribution, and frequently likely to occur together. The most important distinction between types of competition is between exploitation competition which is the result of competitors depleting patches and interference competition which may take several forms. Suggested forms of interference have been time wasted in interactions, effects of forager density on resources (such as disturbing prey) and kleptoparasitism.

It is unlikely that a 'grand unified theory' of competitive distributions will ever be possible since it is difficult to assess the relative importance of the numerous factors involved. Nevertheless, the development of individual level models has provided numerous insights into short term (i.e. less than a single generation) population dynamics. It now remains for ecologists to combine classical population dynamics with these behavioural models.

\section{ACKNOWLEDGEMENTS}

I would like to thank Geoff Parker and Dave Thompson for numerous valuable comments on the manuscript, Bill Sutherland and Kate Lessells for suggestions on content and NERC studentship GT4/91/TLS/30 for funding.

\section{REFERENCES}

Abrahams, M.V. (1986). Patch choice under perceptual constraints: a cause for departures from the IFD. Behav. Ecol. Sociobiol. 19, 409-415. 
Alatalo, R.V., Höglund, J., Lundberg, A. and Sutherland, W.J. (1992). Evolution of black grouse leks: Female preferences benefit males in larger leks. Behav. Ecol. 3, 53-59.

Andren, H. (1990). Despotic distribution, unequal reproductive success, and population regulation in the Jay Garrulus glandarius L. Ecology 71, 1706-1803.

Barnard, C.J. and Brown, C.A.J. (1982). The effects of prior residence, competitive ability and food availability on the outcome of interactions between shrews (Sorex araneus L.) Behav. Ecol. Sociobiol. 10, 307-312.

Bernstein, C., Kacelnik, A. and Krebs, J.R. (1988). Individual decisions and the distribution of predators in a patchy environment. J. Anim. Ecol. 57, 1007-1026.

Bernstein, C. Kacelnik, A. and Krebs, J.R. (1991). Individual decisions and the distribution of predators in a patchy environment II. The influence of travel costs and structure of the environment. J. Anim. Ecol. 60, 205-225.

Borgia, G. (1980). Sexual competition in Scatophaga stercoraria: size- and densityrelated changes in male ability to capture females. Behaviour 75, 185-206.

Brown, J.L. (1969). The buffer effect and productivity in tit populations. Am. Nat. 103, 347-354.

Bulmer, M.G. (1983). Models for the evolution of protandry in insects. Theor. Pop. Biol. 23, 324-322.

Buxton, N.E. (1981). The importance of food in the determination of the winter flock sites of the shelduck. Wildfowl 32, 79-87.

Cézilly, F. and Boy, V. (1991). Ideal free distribution and individual decision rules: a Bayesian approach. Acta. Ecol. 12, 403-410.

Courtney, S.P. and Parker, G.A. (1985). Mating behaviour of the tiger blue butterfly (Tarucus theophrastus) competitive mate-searching when not all females are captured. Behav. Ecol. Sociobiol. 17, 213-221. 
Croy, M.I. and Hughes, R.N. (1991). Effects of food supply, hunger, danger and competition on choice of foraging location by the fifteen-spined stickleback, Spinachia spinachia L. Anim. Behav. 42, 131-139.

Cuthill, I.C., Kacelnik, A., Krebs, J.R., Haccou, P. and Iwasa, Y. (1990). Starlings exploiting patches: the effect of recent experience on foraging decisions. Anim. Behav. 40, 625-640.

Davies, N.B. (1978). Territorial defence in the speckled wood butterfly (Pararge aegaria), the resident always wins. Anim. Behav. 26, 138-147.

Davies, N.B. and Halliday, T.R. (1979). Competitive mate searching in common toads, Bufo bufo. Anim. Behav. 27, 1253-1267.

Davies, N.B. and Houston, A.I. (1984). Territory economics. In: Behavioural Ecology: An Evolutionary Approach, 2nd edition (Ed. by J.R. Krebs and N.B. Davies), pp. 148-169. Blackwell. Oxford.

DeVries, D.R., Stein, R.A. and Chesson, P.L. (1989). Sunfish foraging among patches: the patch -departure decision. Anim. Behav. 37, 455-464.

van Duren, L.A. and Glass, C.W. (1992). Choosing where to feed: the influence of competition on feeding behaviour of cod, Gadus morhua L. J. Fish. Biol. 41, 463471.

Fagen, R. (1987). A generalised habitat matching rule. Evol. Ecol. 1, 5-10.

Fortier, L. and Harris, R.P. (1989). Optimal foraging and density dependent competition in marine fish larvae. Marine Ecology 51, 19-33.

Fretwell, S.D. (1972). Populations in a seasonal environment. Princeton University Press, Princeton, New Jersey.

Fretwell, S.D. and Lucas, H.J. Jr. (1970). On territorial behaviour and other factors influencing habitat distribution in birds. Acta. Biotheor. 19, 16-36.

Gillis, D.M. and Kramer, D.L. (1987). Ideal interference distributions: population density and patch use by zebrafish. Anim. Behav. 35, 1875-1882. 
Godin, J.G.J. and Keenleyside, M.H.A. (1984). Foraging on a patchily distributed prey by a cichlid fish (Teleosti Cichlidae): A test of the IFD theory. Anim. Behav. 32, 120-131.

Goss-Custard, J.D. (1970). The responses of Redshank (Tringa totanus (L.)) to spatial variations in the density of their prey. J. Anim. Ecol. 39, 91-113.

Goss-Custard, J.D. (1980). Competition for food and interference amongst waders. Ardea 68, 31-52.

Goss-Custard, J.D., Clarke, R.T. and Durell, S.E.A.Le V.Dit. (1984). Rate of food intake and aggression of oystercatchers Haematopus ostralegus on the most and least preferred mussel Mytilus edulis beds of the Exe estuary. J. Anim. Ecol. 53, 233-245.

Goss-Custard, J.D., Caldow, R.W.G. and Clarke, R.T. (1992). Correlates of the density of foraging oystercatchers Haematopus ostralegus at different population sizes. J. Anim. Ecol. 61, 159-173.

Goss-Custard, J.D. and Durell, S.E.A.Le V.Dit. (1982). Use of mussel Mytilus edulis beds by Oystercatchers Haematopus ostralegus according to age and population size. J. Anim. Ecol. 51, 543-554.

Gotceitas, V. and Colgan, P. (1991). Assessment of patch suitability and ideal free distribution: the significance of sampling. Behaviour 119, 65-76.

Green, R.F. (1980). Bayesian birds: a simple example of Oaten's stochastic model of optimal foraging. Theor. Pop. Biol. 18, 244-256.

Harley, C.B. (1981). Learning the evolutionarily stable strategy. J. Theor. Biol. 89, 611-633.

Harper, D.G.C. (1982). Competitive foraging in mallards "ideal free" ducks. Anim. Behav. 30, 575-584.

Harris, V.A. (1964). The life of the Rainbow Lizard. Hutchinson Tropical Monographs. Hutchinson and Co. London. $174 \mathrm{pp}$. 
Hassel, M.P. and Varley, G.C. (1969). New inductive model for insect parasites and its bearing on biological control. Nature 223. 1133-1136.

Hassel, M.P. and May, R.M. (1973). Stability in insect host parasite models. $J$. Anim. Ecol. 42, 693-726.

Herrnstein, R.J. (1970). On the law of Effect. J. Exp. Anim. Behav. 21, 159-164.

Holling, C.S. (1959). Some characteristics of simple types of predation and parasitism. Can Entomol. 91, 395-398.

Holling, C.S. (1966). The functional response of predators to prey density and its role in mimicry and population regulation. Mem. Entomol. Soc. Can. 45, 5-60.

Holmgren, N. In press. The ideal free distribution of unequal competitors predictions from a behaviour based functional response. J. Anim. Ecol.

Houston, A.I. and McNamara, J.M. (1988). The IFD when competitive abilities differ: approach based on statistical mechanics. Anim. Behav. 36, 166-174.

Houston, A.I. and McNamara, J.M. (1990). State-dependent ideal free distributions. Evol. Ecol. 4, 298-311.

Houston, A.I., McNamara, J.M. and Milinski, M. In press. The distribution of animals between resources: a compromise between equal numbers and equal intake rates. Anim. Behav.

Houston, A.I. and Sumida, B.H. (1987). Learning rules, matching and frequency dependence. J. Theor. Biol. 126, 289-308.

Hunte, W. Myers, R.A. Doyle, R.W. (1985). Bayesian mating decisions in an amphipod Gammarus lawrencianus Bousfield. Anim. Behav. 33, 366-372.

Huxley, J.S. (1934). A natural experiment on the territorial instinct. Brit. Birds. 27, 270-277. 
Inman, A.J. (1990). Group foraging in starlings: Distributions of unequal competitors. Anim. Behav. 40, 801-810.

Iwasa, Y., Higashi, M. and Yamamura, N. (1981). Prey distribution as a factor determining the choice of optimal foraging strategy. Am. Nat. 117, 710-723.

Iwasa, Y., Odendaal, F.J., Murphy, D.D., Ehrlich, P.R. and Launer, A.E. (1983). Emergence patterns in male butterflies: a hypothesis and a test. Theor. Pop. Biol. 23, 363-379.

Jakobsen, P.J. and Johnsen, G.H. (1987). Behavioural response of the water flea Daphnia pulex to a gradient of food concentration. Anim. Behav. 35, 1891-1895.

Järvi, T. and Pettersen, J.H. (1991). Resource sharing in Atlantic salmon: A test of distribution models on sexually mature and immature parr. Nord. J. Fresh. Res. 66, 89-97.

Kennedy, M. and Gray, R.D. (1993). Can ecological theory predict the distribution of foraging animals? A critical analysis of experiments on the ideal free distribution. Oikos 68, 158-166.

Kacelnik, A. and Krebs, J.R. (1985). Learning to exploit patchily distributed food. In: Behavioural Ecology - ecological consequences of adaptive Behaviour (Ed. by R.M. Sibly and R.H. Smith), pp. 189-205. Blackwell. Oxford.

Korona, R. (1989). Ideal free distribution of unequal competitors can be determined by the form of competition. J. Theor. Biol. 138, 347-352.

Korona, R. (1990). Travel costs and the IFD of ovipositing female flour beetles, Tribolium confusum.. Anim. Behav. 40, 186-187.

Lamb, A.E. and Ollason, J.G. (1993). Foraging wood-ants Formica aquilonia Yarrow (Hymenoptera, Formicidae) tend to adopt the ideal free distribution. Behav Proc. 28, 189-198.

Lessells, C.M. In press. Putting resource dynamics into continuous input ideal free distribution models. Anim. Behav. 
Marschall, E.A. Chesson, P.L. and Stein, R.A. (1989). Foraging in a patchy environment: prey-encounter rate and residence time distributions. Anim. Behav. 37, 444-454.

Maynard Smith, J. (1982). Evolution and the theory of Games. Cambridge University Press, Cambridge.

McNamara, J.M. (1982). Optimal patch use in a stochastic environment. Theor. Pop .Biol. 21, 269-288.

McNamara, J.M. and Houston, A.I. (1990). State dependent ideal free distributions. Evol .Ecol. 4, 298-311.

McNamara, J.M. and Houston, A.I. (1992). Risk sensitive foraging: A review of the theory. Bull. Math. Biol. 54, 355-378.

Messier, F., Virgl, J.A. and Marinelli, L. (1990). Density-dependent habitat selection in muskrats: a test of the ideal free distribution model. Oecologia 84, 380385 .

Milinski, M. (1979). An evolutionary stable feeding strategy in sticklebacks. $Z$ Tierpyschol. 51, 36-40.

Milinski, M. (1984). Competitive resource sharing: an experimental test of a learning rule for evolutionarily stable strategies. Anim. Behav. 32, 233-242.

Milinski, M. (1986). A review of competitive resource sharing under constraints in Sticklebacks. J Fish Biol. 29, 1-14

Milinski, M. In press. Ideal free theory predicts more than only input matching - a critique of Kennedy and Gray's review. Oikos.

Milinski, M. and Parker, G.A. (1991). Competition for resources. In: Behavioural ecology an evolutionary approach, vol 3. (Ed. by J.R. Krebs and N.B. Davies), pp. 137-168. Blackwell. Oxford.

Milinski, M. and Regelmann, K. (1985). Fading short-term memory for patch quality in sticklebacks. Anim. Behav. 33, 678-680. 
Møller, A.P. (1991). Clutch size, nest predation, and distribution of avian unequal competitors in a patchy environment. Ecology 72, 1336-1349.

Monaghan, P. 1980. Dominance and dispersal between feeding sites in the Herring gull (Larus argentatus). Anim. Behav. 28, 521-527.

Morris, D.W. (1989). Density dependent habitat selection: Testing the theory with fitness data. Evol Ecol. 3, 80-94.

Nishida, T. Spatial relationships between mate acquisition probability and aggregation size in a gregarious coreid bug, (Colpula lativentris): A case of the ideal free distribution under perceptual constraints. Res. Pop. Ecol. 35, 45-56.

Oaten, A. (1977). Optimal foraging in patches: A case for stochasticity. Theor. Pop. Biol. 18, 244-256.

Orians, G.H. 1969. On the evolution of mating systems in birds and mammals. Am. Nat. 103, 589-603.

Parker, G.A. (1970). The reproductive behaviour and the nature of sexual selection in Scatophaga stercoraria L. II. The fertilization rate and the spatial and temporal relationships of each sex around the site of mating and oviposition. J. Anim. Ecol. 39, 205-228.

Parker, G.A. (1974). The reproductive behaviour and the nature of sexual selection in Scatophaga stercoraria L. IX. Spatial distribution of fertilization rates and evolution of mate search strategy within the reproductive area. Evolution. 28, 93108.

Parker, G.A. (1978). Searching for mates. In: Behavioural Ecology: An Evolutionary Approach, 1st edn. (Ed. by J.R. Krebs and N.B Davies), pp. 214-244. Blackwell Scientific Publications, Oxford.

Parker, G.A. (1982). Phenotype-limited evolutionarily stable strategies. In: Current problems in sociobiology. (Ed. by Kings college sociobiology group). Cambridge university press. Cambridge. 
Parker, G.A. and Courtney, S.P. (1983). Seasonal incidence: adaptive variation in the timing of life history stages. J. Theor. Biol. 105, 147-155.

Parker, G.A. and Sutherland, W.J. (1986). Ideal free distributions when individuals differ in competitive ability: Phenotype limited ideal free models. Anim. Behav. 34, 1222-1242.

Patterson, I.J. (1985). Limitation of breeding density through territorial behaviour: experiments with convict cichlids, Cichlasoma nigrofasciatum. In: Behavioural Ecology - ecological consequences of adaptive Behaviour (Ed. by R.M. Sibly and R.H. Smith), pp. 393-405. Blackwell. Oxford.

Perusse, D. and Lefebvre, L. (1985). Grouped sequential exploitation of food patches in a flock feeder, the feral pigeon. Behav. Proc. 11, 39-52.

Pimm, S.L. Rosenzweig, M.L. Mitchell, W. (1985). competition and food selection: field tests of a theory. Ecology 66, 798-807.

Pitcher, T.J. and House, A.C. (1987). Foraging rules for group feeders: Area copying depends upon food density in shoaling goldfish. Ethology 76, 161-167.

Power, M.E. (1983). Grazing response of tropical freshwater fishes to different scales of variation in their food. Env. Biol .Fish. 9, 103-115.

Power, M.E. (1984). Habitat Quality and the distribution of algae-grazing catfish in a Panamanian stream. J. Anim. Ecol. 53, 357-374.

Pulliam, H.R. and Caraco, T. (1984). Living in groups: is there an optimal group size? In: Behavioural Ecology and Evolutionary approach, 2nd edition (Ed. by J.R. Krebs and N.B. Davies), pp. 122-147. Blackwell Scientific Publications, Oxford.

Regelmann, K. (1984). Competitive resource sharing- a simulation model. Anim. Behav. 32, 227-232.

Rosenzweig, M.L. (1974). On the evolution of habitat selection. In: Proceedings of the First International Congress of Ecology pp 401-404. Centre for Agricultural Publishing and Documentation, Wageningen, Netherlands. 
Rosenzweig, M.L. (1981). A theory of habitat selection. Ecology 62, 327-335.

Rosenzweig, M.L. (1986). Hummingbird isolegs in an experimental system. Behav. Ecol. Sociobiol. 19, 313-322.

Ross, S. M. (1983). Introduction to Stochastic Dynamic Programming. Academic Press, New York.

Schwinning, S. and Rosenzweig, M.L. (1990). Periodic oscillations in an ideal-free predator-prey distribution. Oikos 59, 85-91.

Sibly, R.M. and McCleery, R.H. (1983). The distribution between feeding sites of herring gulls breeding at Walney island. U.K. J. Anim. Ecol. 52, 51-68.

Sih, A. (1984). The behavioral response race between predator and prey. Am. Nat. 123, 143-150.

Stephens, D.W. and Krebs, J.R. (1986). Foraging theory. Princeton University Press, Princeton, New Jersey.

Sutherland, W.J. (1982). Spatial variation in the predation of cockles by oystercatchers at Traeth Melynog, Anglesey. II. The pattern of mortality. J. Anim. Ecol. 51, 491-500.

Sutherland, W.J. (1983). Aggregation and the "ideal free" distribution. J. Anim. Ecol. 52, 821-828.

Sutherland, W.J. and Allport, G.A. (1994). A spatial depletion model of the interaction between bean geese and wigeon with the consequences for habitat management. J. Anim. Ecol. 63, 51-59.

Sutherland, W.J. and Parker, G.A. (1985). Distribution of unequal competitors. In: Behavioural Ecology - ecological consequences of adaptive Behaviour (Ed. by R.M. Sibly and R.H. Smith), pp. 255-274. Blackwell. Oxford.

Sutherland, W.J. and Parker, G.A. (1992). The relationship between continuous input and interference models of ideal free distributions with unequal competitors. Anim. Behav. 44, 345-355. 
Sutherland, W.J., Townsend, C.R. and Patmore, J.M. (1988). A test of the ideal free distribution with unequal competitors. Behav. Ecol. Sociobiol. 23, 51-53.

Talbot, A.J. and Kramer, D.L. (1986). Effects of food and oxygen availability on habitat selection by guppies in a laboratory environment. Can. J. Zool. 64, 88-93.

Thompson, D.B.A. (1981). Feeding behaviour of wintering shelduck in the Clyde Estuary. Wildfowl 32, 88-98.

Thompson, D.B.A. (1984). Foraging economics in flocks of plovers and gulls. Unpublished $\mathrm{PhD}$ thesis, University of Nottingham.

Thornhill, R. (1980). Sexual selection within mating swarms of the lovebug, Plecia nearetica (Diptera: Bibionidae). Anim. Behav. 28, 405-412.

Tregenza, T. (1994). Common misconceptions in applying the ideal free distribution. Anim. Behav. 47, 485-487.

Valone, T.J. (1989). Group foraging, public information, and patch estimation. Oikos 56, 357-363.

Valone, T.J. (1991). Bayesian and prescient assessment : foraging with pre-harvest information. Anim. Behav. 41, 569-577.

Valone, T.J. (1992). Information for patch assessment: a field investigation with black-chinned hummingbirds. Behav. Ecol. 3, 211-222.

Valone, T.J. and Brown, J.S. (1989). Measuring patch assessment abilities of desert granivores. Ecology 70, 1800-1810.

Whitham, T.B. (1980). The theory of habitat selection: examined and extended using Pemphigus aphids. Am. Nat. 115, 449-466.

Zwarts, L. and Drent, R.H. (1981). Prey depletion and the regulation of predator density: oystercatchers (Haematopus ostralegus ) feeding on mussels (Mytilus edulis ). Feeding and Survival Strategies of Estuarine Organisms (Ed. by N.V. Jones and W.J.Wolff), pp. 193-216. Plenum Publishing Corporation, London. 